\title{
Relèvements de schémas et algèbres de Monsky-Washnitzer : théorèmes d'équivalences et de pleine fidélité II
}

\author{
JeAn-Yves Etesse (*)
}

\section{Sommaire.}

0 . Introduction

1. Généralités

2. Des équivalences de catégories

3. Schémas formels et relèvements de schémas

3.1. Cas des morphismes finis

3.2. Cas des morphismes projectifs et des intersections complètes

\section{Introduction.}

Cet article qui fait suite à [Et 1] en étend les résultats.

Pour faciliter l'exposé, on supposera dans cette introduction que $\mathcal{V}$ est un anneau excellent de caractéristique $0, I \varsubsetneqq \mathcal{V}$ un idéal et $A$ est une $\mathcal{V}$ algèbre lisse.

$\mathrm{Au} \S 1$ on développe un formalisme qui aboutira au $\S 2$ :

- à préciser les liens entre le complété faible $A^{\dagger}$ de $A$ et le séparé complété $I$-adique $\hat{A}$ de $A$;

- et à généraliser l'équivalence de catégories de [Et 1] entre $A^{\dagger}$-schémas finis étales et $\hat{A}$-schémas finis étales.

Par exemple, en s'appuyant sur le caractère hensélien du couple $\left(A^{\dagger}, I A^{\dagger}\right)$, on montre [théo (2.2)] que $A^{\dagger}$ est intégralement fermé dans $\hat{A}$, ce qui est l'analogue d'un théorème de Bosch, Dwork et Robba [Bo-Dw-Ro].

(*) Indirizzo dell'A.: (CNRS - IRMAR, Université de Rennes 1, Campus de Beaulieu - 35042 Rennes Cedex France).

E-mail : Jean-Yves.Etesse@univ-rennes1.fr 
Parmi les généralisations de [Et 1] citons les suivantes:

- On étend la pleine fidélité du foncteur naturel $\left\{A^{\dagger}\right.$-schémas étales $\} \longrightarrow\{\hat{A}$-schémas étales $\}$ du cas $A$ normal, de dimension $\leqslant 1$ de [Et 1 , théo 15] au cas d'une $\mathcal{V}$-algèbre $A$ de type fini quelconque [théo (2.4)] grâce encore au caractère hensélien du couple $\left(A^{\dagger}, I A^{\dagger}\right)$;

- par restriction, le foncteur précédent induit une équivalence de catégories $\left\{A^{\dagger}\right.$-schémas finis étales $\} \longrightarrow\{\hat{A}$-schémas finis étales $\}$ prouvée dans [Et 1, théo 15]. Ici nous explicitons un foncteur quasi-inverse : si $C$ est une $\hat{A}$-algèbre finie étale il suffit, grâce au [théo (2.2)] précédent, de prendre la fermeture intégrale de $A^{\dagger}$ dans $C$ [théo (2.4)];

- lorsque $\mathcal{V}$ est normal et que $(\mathcal{V}, I \mathcal{V})$ est un couple hensélien, on étend entre autres [cor (3.1.4)] cette dernière équivalence de catégories à une équivalence de catégories $\left\{A^{\dagger}\right.$-schémas finis et formellement lisses sur $\mathcal{V}\} \longrightarrow\{\hat{A}$-schémas finis et formellement lisses sur $\mathcal{V}\}$.

Le $§ 3$ rassemble des résultats de relèvements de schémas, de la caractéristique $p>0$ à la caractéristique 0 . Sur une base affine, les cas les plus importants pour les applications (cf plus bas) seront celui d'un morphisme fini [théo (3.1.3)], d'un morphisme fini étale [théo (3.1.1)] ( resp. et galoisien [cor (3.1.6)]), d'un morphisme projectif lisse [théo (3.2.1)] et son corollaire [cor (3.2.6)] pour les intersections complètes.

En plus des généralisations de [Et 1] mentionnées ci-dessus, les relèvements du $\S 3$ nous servent dans deux contextes :

- les relèvements de morphismes projectifs lisses dans le cas des intersections complètes [cor (3.2.6)] permettent d'établir la surconvergence d'images directes en cohomologie rigide [Et 3] (cf [Et 6, chap II]);

- les relèvements de morphismes finis permettent de relever le Frobenius pour les $F$-isocristaux surconvergents et de prouver la surconvergence d'images directes de ceux-ci [Et 5] (cf [Et 6, chap IV]).

\section{Généralités.}

\subsection{Notations.}

Tous les anneaux considérés dans cet article sont (sauf mention du contraire) commutatifs et unitaires.

Soient $\mathcal{V}$ un anneau noethérien, $I \subsetneq \mathcal{V}$ un idéal, $A$ une $\mathcal{V}$-algèbre telle que l'anneau $A$ soit noethérien et $I A \neq A$. On note $\hat{A}$ le séparé complété $I$ adique de $A, A_{n}=A / I^{n+1} A$ et $A^{\dagger} \subset \hat{A}$ le complété faible de $A$ au-dessus de 
la paire $(\mathcal{V}, I)[\mathrm{M}-\mathrm{W}, \S 1]$ : on désignera toujours par un indice ( $)_{0}$ la réduction $\bmod I$ d'une $\mathcal{V}$-algèbre ou d'un $\mathcal{V}$-morphisme.

Si $B$ est une $\mathcal{V}$-algèbre, on dit que $B$ est faiblement complète de type fini (f.c.t.f. en abrégé) si $B$ est la complétée faible d'une $\mathcal{V}$-algèbre de type fini ; une telle algèbre $B$ est appelée "w.c.f.g." dans la terminologie de [M-W, § 2].

Considérons la partie multiplicative $T=1+I A$ de $A$; notons $A_{T}=T^{-1} A$ et $(\tilde{A}, \tilde{I})$ le hensélisé de $(A, I A)$ au sens de Raynaud [R, déf 4 p. 24]: rappelons qu'on a supposé $I A \neq A$, si bien que $0 \notin T$; sinon les anneaux $A_{T}, \tilde{A}, \hat{A}$ et $A^{\dagger}$ seraient égaux à $\{0\}$.

On rappelle [Et 1] que si $A$ est une $\mathcal{V}$-algèbre de type fini, alors il existe des morphismes canoniques $A_{T} \rightarrow \tilde{A} \rightarrow A^{\dagger} \rightarrow \hat{A}$ tous fidèlement plats et que tous ces anneaux ont même séparé complété $I$-adique égal à $\hat{A}$.

Proposition (1.1). Soient $A, \mathcal{A}, \mathcal{B}$ des anneaux noethériens munis de morphismes

$$
A \stackrel{\varphi_{1}}{\longrightarrow} \mathcal{A} \stackrel{\varphi_{2}}{\longrightarrow} \mathcal{B} \stackrel{\varphi_{3}}{\longrightarrow} \hat{A}
$$

avec $\varphi_{3}$ fidèlement plat. On suppose que Spec $\hat{A} \longrightarrow$ Spec $A$ est un morphisme normal (resp. régulier) [EGA IV, (6.8.1)] : cette dernière hypothèse est vérifiée si $A$ est excellent. Alors:

(1) Le morphisme

$$
h=\operatorname{Spec}\left(\varphi_{2} \circ \varphi_{1}\right): \operatorname{Spec} \mathcal{B} \rightarrow \operatorname{Spec} A
$$

est normal (resp. régulier).

(2) Si de plus $\varphi_{2}$ est plat, alors

$$
f=\operatorname{Spec}\left(\varphi_{2}\right): \operatorname{Spec} \mathcal{B} \rightarrow \operatorname{Spec} \mathcal{A}
$$

est un morphisme normal.

(3) Si $\varphi_{2}$ est plat et $(\mathcal{A}, I \mathcal{A})$ est un couple hensélien tel que $\hat{\mathcal{A}} \simeq \hat{A}$, alors

$$
f: \operatorname{Spec} \mathcal{B} \rightarrow \operatorname{Spec} \mathcal{A}
$$

est un morphisme normal à fibres géométriquement intègres.

(4) Si $\varphi_{2}$ est plat, $(\mathcal{A}, I \mathcal{A})$ est un couple hensélien tel que $\hat{\mathcal{A}} \simeq \hat{A}$ et $\mathcal{A}$ est réduit, alors $\mathcal{A}$ est intégralement fermé dans $\mathcal{B}$ et dans $\hat{A}$.

DÉmonstration. Le (1) résulte de [EGA IV, (6.5.4) (i) (resp. (6.5.2) (i))]. Pour le (2) notons $g=\operatorname{Spec} \varphi_{1}: \operatorname{Spec} \mathcal{A} \rightarrow \operatorname{Spec} A$. Soit $q \in \operatorname{Spec} \mathcal{A}$ et $k^{\prime}$ une extension finie du corps résiduel $k(\mathfrak{q})$ : il s'agit de montrer que

$$
f^{-1}(\mathfrak{q})_{k^{\prime}}=\operatorname{Spec}\left(k^{\prime} \otimes_{\mathcal{A}} \mathcal{B}\right)
$$


est normal [EGA IV, (6.8.1)]. Comme Spec $k^{\prime}$ est normal et $h$ un morphisme normal, on sait par [EGA IV, (6.14.1)] que Spec $\left(k^{\prime} \otimes_{A} \mathcal{B}\right)$ est normal.

Considérons les applications

$$
\begin{aligned}
& k^{\prime} \otimes_{\mathcal{A}} \mathcal{B} \stackrel{\psi}{\longrightarrow} k^{\prime} \otimes_{A} \mathcal{B} \simeq k^{\prime} \otimes_{\mathcal{A}}\left(\mathcal{A} \otimes_{A} \mathcal{B}\right) \stackrel{\varphi}{\longrightarrow} k^{\prime} \otimes_{\mathcal{A}} \mathcal{B} \\
& x \otimes b \longmapsto x \otimes\left(1_{\mathcal{A}} \otimes b\right) \\
& x \otimes(a \otimes b)
\end{aligned}
$$

clairement $\varphi \circ \psi=I d$. Pour $\mathfrak{p} \in \operatorname{Spec}\left(k^{\prime} \otimes_{\mathcal{A}} \mathcal{B}\right), \quad \mathfrak{m}:=\varphi^{-1}(\mathfrak{p}) \quad$ on $\quad$ a $\mathfrak{p}=\psi^{-1}\left(\varphi^{-1}(\mathfrak{p})\right)=\psi^{-1}(\mathfrak{m})$ et $\psi$ et $\varphi$ induisent des morphismes

$$
\left(k^{\prime} \otimes_{\mathcal{A}} \mathcal{B}\right)_{\mathfrak{p}} \stackrel{\psi^{\prime}}{\longrightarrow}\left(k^{\prime} \otimes_{A} \mathcal{B}\right)_{\mathfrak{m}} \stackrel{\varphi^{\prime}}{\longrightarrow}\left(k^{\prime} \otimes_{\mathcal{A}} \mathcal{B}\right)_{\mathfrak{p}}
$$

dont le composé est encore l'identité.

Par hypothèse $\mathcal{D}:=\left(k^{\prime} \otimes_{A} \mathcal{B}\right)_{\mathfrak{m}}$ est intégralement clos, de corps des fractions noté $L$; en particulier $\mathcal{C}:=\left(k^{\prime} \otimes_{\mathcal{A}} \mathcal{B}\right)_{\mathfrak{p}}$ est intègre : notons $K$ son corps des fractions. Il s'agit de montrer que $\mathcal{C}$ est intégralement clos: soit $x \in K$ un élément entier sur $\mathcal{C}$, annulé par le polynôme $R(X)=$ $=X^{n}+\sum_{i=0}^{n-1} a_{i} X^{i}, a_{i} \in \mathcal{C}$. L'injection $\psi^{\prime}$ induit une injection:

$$
\psi^{\prime \prime}: K \hookrightarrow L ;
$$

puisque $\mathcal{D}$ est intégralement clos on a $\psi^{\prime \prime}(x) \in \mathcal{D}$ et $x_{1}:=\varphi^{\prime}\left(\psi^{\prime \prime}(x)\right) \in \mathcal{C}$ est racine de $R(X)$ : en effet $\psi^{\prime \prime}$ induit $\tilde{\psi}^{\prime \prime}: K[X] \rightarrow L[X], R(X) \mapsto \tilde{R}(X) \in$ $\in \mathcal{D}[X]$ et $\varphi^{\prime}$ induit $\tilde{\varphi}^{\prime}: \mathcal{D}[X] \rightarrow \mathcal{C}[X], \quad \tilde{R}(X) \mapsto R(X)$. D'où $R(X)=$ $=\left(X-x_{1}\right) R_{1}(X)$ avec $R_{1}(X) \in \mathcal{C}[X]$. Si $x=x_{1}$ on a terminé, sinon $x$ est racine de $R_{1}(X)$ et on itère : finalement $x \in \mathcal{C}$, donc $\mathcal{C}$ est intégralement clos, i.e. $f^{-1}(\mathfrak{q})_{k^{\prime}}$ est normal.

Pour le (3), on sait par le (2) que le morphisme $g$ : Spec $\hat{\mathcal{A}}=\operatorname{Spec} \hat{A} \rightarrow$ $\rightarrow$ Spec $\mathcal{A}$ est normal, donc ses fibres sont géométriquement intègres par un théorème de Raynaud [R, théo $3, \mathrm{p} .126]$. Or $\hat{A}$ est une $\mathcal{B}$-algèbre fidèlement plate, donc les fibres de $f$ sont aussi géométriquement intègres.

Pour le (4), le théorème de Raynaud [loc. cit.] prouve que $\mathcal{A}$ est intégralement fermé dans $\hat{A}$ : comme $\varphi_{3}$ est injectif, $\mathcal{A}$ est aussi intégralement fermé dans $\mathcal{B}$.

Pour la commodité des références nous avons rassemblé ci-après quelques lemmes qui résultent des EGA.

Lemme (1.2). Soient $\mathcal{A}$ et $\mathcal{B}$ deux anneaux noethériens tels que $\mathcal{B}$ soit une $\mathcal{A}$-algèbre. Les propriétés (i) et (ii) ci-après sont équivalentes: 
(i) Spec $\mathcal{B} \rightarrow$ Spec $\mathcal{A}$ est un morphisme régulier.

(ii) Pour tout $\mathfrak{q} \in \operatorname{Spec} \mathcal{A}$ et tout $\mathfrak{p} \in \operatorname{Spec} \mathcal{B}$ au-dessus de $\mathfrak{q}$, le morphisme $\mathcal{A}_{\mathfrak{q}} \rightarrow \mathcal{B}_{\mathfrak{p}}$ est formellement lisse pour les topologies préadiques respectives (i.e. définies par q $\mathcal{A}_{\mathfrak{q}}$ et $\mathfrak{p} \mathcal{B}_{\mathfrak{p}}$ respectivement).

DÉmONSTRATION. En utilisant la définition d'un morphisme régulier [EGA IV, (6.8.1)], l'équivalence résulte de [EGA $O_{I V},(22.5 .8)$ et (19.7.1)].

Lemme (1.3). Soient $\mathcal{A}$ et $\mathcal{B}$ deux anneaux noethériens tels que $\mathcal{B}$ soit une $\mathcal{A}$-algèbre. Si Spec $\mathcal{B} \rightarrow$ Spec $\mathcal{A}$ est formellement lisse pour les topologies discrètes, alors c'est un morphisme régulier.

DÉmonstration. Si $\mathcal{B}$ est une $\mathcal{A}$-algèbre formellement lisse pour les topologies discrètes [EGA $\left.O_{I V},(19.3 .1)\right]$, alors pour tout $\mathfrak{q} \in \operatorname{Spec} \mathcal{A}$ et tout $\mathfrak{p} \in \operatorname{Spec} \mathcal{B}$ au-dessus de $\mathfrak{q}$, le morphisme

$$
\mathcal{A}_{\mathfrak{q}} \rightarrow \mathcal{B}_{\mathfrak{p}}
$$

est formellement lisse pour les topologies discrètes [EGA $\left.O_{I V},(19.3 .5)(i v)\right]$, donc aussi pour les topologies préadiques sur $\mathcal{A}_{\mathfrak{q}}$ et $\mathcal{B}_{\mathfrak{p}}\left[\mathrm{EGA} O_{I V}\right.$, (19.3.8)] ; d'où la conclusion par le lemme (1.2).

Lemme (1.4). Soient $\mathcal{A}$ un anneau, $\mathcal{B}$ une $\mathcal{A}$-algèbre et $\mathcal{J} \subset \mathcal{A}$ un idéal. Si Spec $\mathcal{B} \rightarrow$ Spec $\mathcal{A}$ est formellement lisse pour les topologies discrètes, alors c'est un morphisme formellement lisse pour les topologies $\mathcal{J}$-adiques $\operatorname{sur} \mathcal{A}$ et $\mathcal{B}$.

DÉmonstration. Via [EGA $\left.O_{I V},(19.3 .8)\right]$.

Lemme (1.5). Soient $\mathcal{A}$ un anneau et $S \subset \mathcal{A}$ une partie multiplicative. Alors

(i) Le morphisme $f: X=\operatorname{Spec}\left(S^{-1} \mathcal{A}\right) \rightarrow \operatorname{Spec} \mathcal{A}=Y$ est formellement étale pour les topologies discrètes.

(ii) En particulier f est régulier si $\mathcal{A}$ est noethérien.

(iii) De plus :

* si $y \in Y \backslash f(X)$, alors $f^{-1}(y)=\phi$

* si $y \in f(X)$, alors $f$ induit un isomophisme

$f^{-1}(y) \stackrel{\sim}{\longrightarrow}$ Spec $k(y)$. 
DÉmonstration. La première assertion n'est autre que [EGA $O_{I V}$, (19.10.3) (ii)]; la deuxième en résulte grâce au lemme (1.3). La dernière assertion est conséquence de [Bour, AC II, § 2, n 5, prop 11].

Lemme (1.6). Soient $\mathcal{A}$ et $\mathcal{B}$ deux anneaux noethériens tels que $\mathcal{B}$ soit une A-algèbre; notons

$$
f: \operatorname{Spec} \mathcal{B} \rightarrow \operatorname{Spec} \mathcal{A}
$$

le morphisme canonique. Alors:

(1) On a les implications:

(i) $f$ est réduit et $\mathcal{A}$ réduit $\Rightarrow \mathcal{B}$ réduit.

(ii) $f$ normal et $\mathcal{A}$ normal $\Rightarrow \mathcal{B}$ normal.

(iii) $f$ régulier et $\mathcal{A}$ régulier $\Rightarrow \mathcal{B}$ régulier.

(2) Sif est fidèlement plat, on a les implications:

(i) $\mathcal{B}$ réduit $\Rightarrow \mathcal{A}$ réduit.

(ii) $\mathcal{B}$ normal $\Rightarrow \mathcal{A}$ normal.

(iii) $\mathcal{B}$ régulier $\Rightarrow \mathcal{A}$ régulier.

DÉMONSTRATION. (1) (i) On utilise la définition d'un morphisme réduit [EGA IV, (6.8.1)] et la caractérisation des schémas noethériens réduits de [EGA IV, (5.8.5)] via les propriétés $\ll R_{0}$ et $S_{1} \gg$ : comme $\mathcal{A}$ vérifie $\ll R_{0}$ et $S_{1} \gg$, il en est de même de $\mathcal{B}$ via [EGA IV, (6.5.3) (ii) et (6.4.2)], donc $\mathcal{B}$ est réduit.

Pour (ii) (resp. (iii)) on utilise [EGA IV, (6.5.4) (ii)] (resp. [EGA IV, (6.5.2) (ii)]).

(2) (i) Le fait qu'un schéma $X$ est réduit s'exprime via les anneaux locaux $\mathcal{O}_{X, x}\left[\right.$ EGA $\left.0_{I},(4.1 .4)\right]$ : le (i) résulte de [EGA IV, (2.1.13)].

Le (ii), c'est [EGA IV, (6.5.4) (i)] et le (iii) c'est [EGA IV, (6.5.2) (i)].

La proposition suivante généralise des assertions de [Et 1, prop 2, prop 11].

Proposition (1.7). Soient A une V-algèbre (resp. une V-algèbre de type fini) telle que $A$ soit un anneau noethérien. Notons $\mathcal{B}$ l'un des anneaux $\tilde{A}, \hat{A}$ (resp. $\left.A^{\dagger}\right)$. Alors

(1) $A_{T}$ et $\mathcal{B}$ sont des anneaux de Zariski.

(2) On a les implications: 
(i) $A$ réduit $\Rightarrow A_{T}$ réduit $\Leftrightarrow \tilde{A}$ réduit.

(ii) A normal $\Rightarrow A_{T}$ normal $\Leftrightarrow \tilde{A}$ normal.

(iii) $A$ régulier $\Rightarrow \hat{A}$ régulier (resp. $\Rightarrow A^{\dagger}$ régulier) $\Rightarrow \tilde{A}$ régulier $\Rightarrow$ $\Rightarrow A_{T}$ régulier.

(iv) A intégralement clos $\Rightarrow A_{T}$ intégralement clos.

(3) $S i f_{T}:$ Spec $\hat{A} \rightarrow$ Spec $A_{T}$ est le morphisme canonique et $f$ le composéf : Spec $\hat{A} \underset{f_{T}}{\longrightarrow}$ Spec $A_{T} \longrightarrow$ Spec A, on a l'implication: $f$ réduit (resp. normal; resp. régulier) $\Rightarrow f_{T}$ réduit (resp. normal; resp. régulier).

(4) (i) Sif est réduit on a les implications:

$A$ réduit $\Rightarrow A_{T}$ réduit $\Leftrightarrow \mathcal{B}$ réduit.

(ii) Sif est normal on a les implications:

A normal $\Rightarrow A_{T}$ normal $\Leftrightarrow \mathcal{B}$ normal.

(iii) Sif est régulier on a les implications:

$A$ régulier $\Rightarrow A_{T}$ régulier $\Leftrightarrow \mathcal{B}$ régulier.

(iv) Sif est normal on a les implications:

$A$ intégralement clos $\Rightarrow A_{T}$ intégralement clos $\Leftrightarrow \mathcal{B}$ intégralement clos.

DÉMONSTRATION.

(1) $A_{T}$ est noethérien [Bour, AC II, $\S 2, n^{\circ} 4$, cor 2 de prop 10], de même que $\tilde{A}$ [R, p. 125] et $\hat{A}$ [Bour, AC III, $\S 3, \mathrm{n}^{\circ} 4$, prop 8]. De plus si $A$ est de type fini sur $\mathcal{V}$, alors $A^{\dagger}$ est noethérien [M-W, theo 2.1]. De plus par [Et $1, \S 1$ ] on a les inclusions $I A_{T} \subset \operatorname{Rad} A_{T}$, $I \tilde{A} \subset \operatorname{Rad} \tilde{A}, I A^{\dagger} \subset \operatorname{Rad} A^{\dagger}, I \hat{A} \subset \operatorname{Rad} \hat{A} ;$ donc les anneaux $A_{T}, \tilde{A}$, $A^{\dagger}$ et $\hat{A}$ sont de Zariski.

(2) Grâce aux lemmes (1.5) et (1.3), l'implication

$A$ réduit (resp. normal; resp. régulier) $\Rightarrow A_{T}$ de même, résulte du [lemme (1.6), (1)] et l'équivalence

$A_{T}$ réduit (resp. normal) $\Leftrightarrow \tilde{A}$ de même,

c'est [R, p. 125].

Si $A$ est régulier, alors $\hat{A}$ l'est [EGA $\mathrm{O}_{I V}$, (17.3.8.1)]: par fidèle platitude de $\hat{A}$ sur $A^{\dagger}, \tilde{A}$ et $A_{T}$, ces derniers sont aussi réguliers [EGA IV, (6.5.2)(i)].

Si $A$ est intégralement clos, rappelons que $0 \notin T$, donc $A_{T}$ est intègre de même corps des fractions que $A$, par suite $A_{T}$ est intégralement clos [Bour, AC V, § 1, $\mathrm{n}^{\circ} 5$, prop 16].

(3) Résulte du [lemme (1.5) (iii)] et de [EGA IV, (6.8.1)]. 
(4) D'après le (2) et le (3) on est ramené à prouver le (4) en remplaçant $A$ par $A_{T}$ et $f$ par le morphisme fidèlement plat $f_{T}$.

Les assertions (i) à (iii) sont fournies par le lemme (1.6).

Pour le (iv) supposons d'abord $\mathcal{B}$ intégralement clos : par fidèle platitude de $\mathcal{B}$ sur $A_{T}$ on en déduit que Spec $A_{T}$ est connexe, normal [EGA IV, (2.1.13)] et noethérien, donc $A_{T}$ est intègre [EGA I, (4.5.6)] et intégralement clos par [Bour, AC II, $\S 3, \mathrm{n}^{\circ} 3$, cor 4 du théo 1 et AC V, $\S 1, \mathrm{n}^{\circ} 2$, cor de prop 8].

Réciproquement supposons $\mathcal{A}:=A_{T}$ intégralement clos: par fidèle platitude de $\hat{A}$ sur $\mathcal{B}$ il nous suffit de montrer que $\hat{A}=\hat{\mathcal{A}}$ est intégralement clos. Puisque $\mathcal{A}$ est intègre, l'idéal $I \mathcal{A}$ est sans torsion, par suite $\mathcal{A}=\bigcap_{\mathfrak{p}} \mathcal{A}_{\mathfrak{p}}$ et $I \mathcal{A}=\bigcap_{\mathfrak{p}} I \mathcal{A}_{\mathfrak{p}}$ où $\mathfrak{p}$ parcourt l'ensemble $M$ des idéaux maximaux de $\mathcal{A}$ [Bour, AC II, § 3, $\mathrm{n}^{\circ} 3$, cor 4 du théo 1]; comme $\mathcal{A} / I \mathcal{A} \simeq A / I A \neq\{0\}$ par hypothèse, il existe donc $\mathfrak{p} \in M$ tel que $I \mathcal{A}_{\mathfrak{p}} \varsubsetneqq \mathcal{A}_{\mathfrak{p}}$. Soit $\mathfrak{p} \in M$ tel que $I \mathcal{A}_{\mathfrak{p}} \varsubsetneqq \mathcal{A}_{\mathfrak{p}}$ : l'idéal $I \mathcal{A}_{\mathfrak{p}}$ est contenu dans le seul idéal maximal $\mathfrak{p} \mathcal{A}_{\mathfrak{p}}$ de $\mathcal{A}_{\mathfrak{p}}$. Or l'inclusion $\mathcal{A} \hookrightarrow \mathcal{A}_{\mathfrak{p}}$ donne l'inclusion $\left.\varphi: \hat{\mathcal{A}} \hookrightarrow \widehat{\left(\mathcal{A}_{\mathfrak{p}}\right.}\right):=\lim _{\breve{r}} \mathcal{A}_{\mathfrak{p}} / I^{n} \mathcal{A}_{\mathfrak{p}}$, où $\widehat{\left(\mathcal{A}_{\mathfrak{p}}\right)}$ est local d'idéal maximal $\mathfrak{p}\left(\widehat{\mathcal{A}_{\mathfrak{p}}}\right)$ [Bour, AC III, $\S 3, \mathrm{n}^{\circ} 4$, prop 8 (ii)]: le schéma Spec $\left(\widehat{\mathcal{A}_{\mathfrak{p}}}\right)$ est connexe et son image par le morphisme dominant

$$
\text { Spec } \varphi: \operatorname{Spec} \widehat{\left(\mathcal{A}_{\mathfrak{p}}\right)} \rightarrow \operatorname{Spec} \hat{\mathcal{A}}
$$

est un connexe dense, donc $\operatorname{Spec} \hat{\mathcal{A}}$ est connexe ; comme $\hat{\mathcal{A}}$ est noethérien (cf (1)) et normal (cf (4) (ii)), il en résulte que $\hat{\mathcal{A}}$ est intègre [EGA I, (4.5.6)] et intégralement clos.

\section{Des équivalences de catégories.}

THÉORÈme (2.1). Soit A une $\mathcal{V}$-algèbre telle que l'anneau $A$ soit noethérien. On suppose que le morphisme canonique Spec $\hat{A} \rightarrow$ Spec A est normal (vrai par exemple si A est excellent). Alors

(1) Le morphisme canonique

$$
f: \operatorname{Spec} \hat{A} \rightarrow \operatorname{Spec} \tilde{A}
$$

est normal, fidèlement plat, à fibres géométriquement intègres.

(2) (i) Si Ã est réduit alors Ã est intégralement fermé dans $\hat{A}$.

(ii) Le (i) est vérifié si A est réduit. 
(iii) On a les équivalences:

$\tilde{A}$ intègre (resp. intégralement clos)

$\Leftrightarrow \hat{A}$ intègre (resp. intégralement clos).

Démonstration. Pour le (1), $\hat{A}$ est le séparé complété $I$-adique de $\tilde{A}$, et $\tilde{A}$ est de Zariski [prop (1.7) (1)], donc $f$ est fidèlement plat [Bour, AC III, $\S 3, \mathrm{n}^{\circ} 5$, prop 9]. L'hypothèse entraîne alors que $f$ est normal à fibres géométriquement intègres via [prop (1.1) (3)], car $(\tilde{A}, \tilde{I})$ est un couple hensélien [R, théo 3, p. 126].

Le (2) (i) résulte de [prop (1.1) (4)], car $(\tilde{A}, \tilde{I})$ est un couple hensélien, et le (2) (ii) provient de [prop (1.7) (2) (i)].

Dans le (2) (iii) l'assertion dans le cas "intégralement clos" est prouvée dans [prop (1.7), (4) (iv)]. Pour le cas "intègre", comme $\tilde{A} \hookrightarrow \hat{A}$ est fidèlement plat, il suffit de prouver que si $\tilde{A}$ est intègre, alors $\hat{A}$ l'est: supposons $\tilde{A}$ intègre, alors $\hat{A}$ est réduit [prop (1.7) (4) (i)]; or les fibres de $f$ sont géométriquement intègres, ainsi Spec $\hat{A}$ est irréductible [EGA IV, (2.3.5) (iii)] et réduit, donc $\hat{A}$ est intègre.

ThÉoRÈme (2.2). Soit A une $\mathcal{V}$-algèbre de type fini; on suppose que le morphisme canonique Spec $\hat{A} \rightarrow$ Spec A est normal (vrai par exemple si $\mathcal{V}$ est excellent). Alors:

(1) Les morphismes canoniques

$$
g: \operatorname{Spec} \hat{A} \longrightarrow \operatorname{Spec} A^{\dagger}, h: \operatorname{Spec} A^{\dagger} \longrightarrow \operatorname{Spec} \tilde{A}
$$

sont normaux, fidèlement plats, à fibres géométriquement intègres.

(2) (i) Si $\tilde{A}$ est réduit, $\tilde{A}$ est intégralement fermé dans $A^{\dagger}$ et dans $\hat{A}$.

(ii) $S i A^{\dagger}$ est réduit, $A^{\dagger}$ (resp. $A_{K}^{\dagger}$ ) est intégralement fermé dans $\hat{A}$ (resp. dans $\widehat{A}_{K}$ ).

(iii) Les hypothèses (i) et (ii) sont satisfaites si A est réduit.

(iv) On a les équivalences:

A intègre (resp. intégralement clos)

$\Uparrow$

$A^{\dagger}$ intègre (resp. intégralement clos)

茫

$\hat{A}$ intègre (resp. intégralement clos).

DÉMONSTRATION.

(1) La preuve pour $g$ est identique à celle du théorème précédent en remarquant que $\left(A^{\dagger}, I A^{\dagger}\right)$ est un couple hensélien [Et 1, théo 3]. 
Le cas de $h$ a été traité dans [prop (1.1) (3)].

(2) Le (i) et le (ii) ont été vus dans le (4) de [prop (1.1)].

Le (iii) provient du (4) (i) de [prop (1.7)].

Dans le (iv) l'assertion dans le cas "intégralement clos" est prouvée dans [prop (1.7) (4) (iv)]. Pour le cas "intègre" les inclusions $\tilde{A} \subset A^{\dagger} \subset \hat{A}$ ramènent la preuve au (2) (iii) du théorème (2.1).

Remarque (2.2.1). Lorsque $\mathcal{V}$ est un anneau de valuation discrète complet et $A$ une $\mathcal{V}$-algèbre de type fini, $A$ est excellent [EGA IV, (7.8.3)]. $\mathrm{Si} A^{\dagger}$ est réduit, le (2) (ii) du théorème (2.2) prouve que $A^{\dagger}$ est intégralement fermé dans $\hat{A}$ : on retrouve ainsi l'analogue du théorème 2 de Bosch, Dwork, Robba de [Bo-Dw-R] lorsque la valuation de $K$ de [loc. cit.] est discrète; cf. aussi [Bo].

Nous sommes maintenant en mesure de prouver les théorèmes (2.3) et (2.4) qui suivent, et qui améliorent le théorème 15 de [Et 1].

ThÉorÈme (2.3). Soit A une A-algèbre telle que l'anneau A soit noethérien. On suppose que le morphisme canonique Spec $\hat{A} \rightarrow$ Spec A est normal (vrai si A est excellent). Alors

(1) Le foncteur $\mathcal{E}$ de la catégorie des $\tilde{A}$-schémas étales dans la catégorie des $\hat{A}$-schémas étales défini par

$$
f: \operatorname{Spec} \hat{A} \rightarrow \operatorname{Spec} \tilde{A}
$$

est pleinement fidèle.

(2) (i) Le foncteur $\mathcal{F}: B \mapsto B \otimes_{\tilde{A}} \hat{A}$ de la catégorie des $\tilde{A}$-algèbres finies étales dans la catégorie des $\hat{A}$-algèbres finies étales défini parf est une équivalence de catégories.

(ii) Si $\tilde{A}$ est réduit (c'est le cas par exemple si A est réduit), le foncteur $\mathcal{G}$ qui à une $\hat{A}$-algèbre finie étale $C$ associe la fermeture intégrale de $\tilde{A}$ dans $C$ est un foncteur quasi-inverse de $\mathcal{F}$.

DÉmonstration. Le (1) et le (2) (i) se démontrent comme le (1) du théorème 15 de [Et 1].

Pour (2) (ii) il suffit, grâce au fait que $\mathcal{F}$ est une équivalence de catégories, de prouver que si $B$ est une $\tilde{A}$-algèbre finie étale, alors $B$ est la fermeture intégrale de $\tilde{A}$ dans $B \otimes_{\tilde{A}} \hat{A} \simeq \hat{B}$.

Supposons $\tilde{A}$ réduit et soit $B$ une $\tilde{A}$-algèbre finie étale : alors $(B, I B)$ est un couple hensélien [R, prop 2 (1) p. 124], $B$ est réduit par le (1) (i) du 
[lemme (1.6)] et Spec $\hat{B} \rightarrow$ Spec $B$ est un morphisme normal [EGA IV, (6.8.3) (iii)]; par le (4) de la [prop (1.1)] on en déduit que $B$ est intégralement fermé dans $\hat{B}$. Comme $B$ est fini sur $\tilde{A}, B$ est bien la fermeture intégrale de $\tilde{A}$ dans $\hat{B}$.

THÉORÈme (2.4). Soit A une $\mathcal{V}$-algèbre de type fini; on suppose que le morphisme canonique Spec $\hat{A} \rightarrow$ Spec A est normal (vrai par exemple si $\mathcal{V}$ est excellent) et on désigne par $(\mathcal{A}, \mathcal{B})$ l'un des couples $\left(\tilde{A}, A^{\dagger}\right),\left(A^{\dagger}, \hat{A}\right)$. Alors

(1) Le foncteur $\mathcal{E}$ de la catégorie des $\mathcal{A}$-schémas étales dans la catégorie des $\mathcal{B}$-schémas étales défini par

$$
f: \operatorname{Spec} \mathcal{B} \rightarrow \operatorname{Spec} \mathcal{A}
$$

est pleinement fidèle.

(2) (i) Le foncteur: $\mathcal{F}: B \mapsto B \otimes_{\mathcal{A}} \mathcal{B}$ de la catégorie des $\mathcal{A}$-algèbres finies étales dans la catégorie des $\mathcal{B}$-algèbres finies étales défini par $f$ est une équivalence de catégories.

(ii) $S i \mathcal{A}$ est réduit (c'est le cas par exemple si A est réduit), le foncteur $\mathcal{G}$ qui à une $\mathcal{B}$-algèbre finie étale $C$ associe la fermeture intégrale de $\mathcal{A}$ dans $C$ est un foncteur quasi-inverse de $\mathcal{F}$.

DÉMONSTRATION.

(1) Ici $f$ est fidèlement plat et quasi-compact, donc universellement submersif [EGA I, (3.9.4) (ii) et (7.3.5)]; de plus les fibres de $f$ sont géométriquement intègres [théo (2.2)]. En vertu de [SGA 1, IX, cor $3.4]$ le foncteur $\mathcal{E}$ défini par $f$ est pleinement fidèle.

(2) Le (i) se montre comme [Et 1, théo 15, 2 (i) et (ii)]. La preuve du (ii) est la même que celle du (2) (ii) du [théorème (2.3)] si l'on rappelle que toute $A^{\dagger}$-algèbre finie $B$ est "f.c.t.f" [Et 1, prop 1] et que $(B, I B)$ est un couple hensélien [loc. cit, théo 3].

REMARque (2.4.1). La partie (1) des théorèmes (2.3) et (2.4) précédents est une généralisation de [EGA IV, (18.9.5)].

\section{Schémas formels et relèvements de schémas.}

3.1 - Cas des morphismes finis.

THÉORÈme (3.1.1). Soit $A$ une $\mathcal{V}$-algèbre normale de caractéristique zéro telle que $A$ soit un anneau noethérien; on suppose $A$ excellent et $0 \notin T:=1+I A$. On note $A_{0}=A / I A$. Alors 
(1) Si $\varphi: S^{\prime} \rightarrow$ Spec $A_{0}=: S$ est un morphisme fini étale, il existe un morphisme fini

$$
\psi: \operatorname{Spec} B \rightarrow \operatorname{Spec} A
$$

relevant $\varphi$, où $B$ est normal (resp. B est intégralement clos si A l'est) et tel que

$$
\psi_{T}: \operatorname{Spec} B_{T} \rightarrow \operatorname{Spec} A_{T}
$$

soit un relèvement fini étale de $\varphi$.

(2) De plus il existe $g_{0} \in A_{0}$ et $g \in A$ relevant $g_{0}$ tels que

$$
\psi_{g}: \text { Spec } B_{g} \rightarrow \text { Spec } A_{g}
$$

soit un relèvement fini étale de

$$
\varphi_{g_{0}}: S_{g_{0}}^{\prime} \rightarrow \operatorname{Spec}\left(A_{0_{g_{0}}}\right) .
$$

(3) Supposons de plus $\mathcal{V}$ excellent, $A$ de type fini sur $\mathcal{V}$ et fixons une présentation

$$
A=\mathcal{V}\left[t_{1}, \ldots, t_{n}\right] /\left(f_{1}, \ldots, f_{r}\right)
$$

Notons $P$ la fermeture projective de Spec $A$ dans $\mathbb{P}_{\mathcal{V}}^{n}, P^{\prime}$ le normalisé de $P$ et $P^{\prime \prime}$ la fermeture intégrale de $P$ dans l'anneau $\mathcal{R}$ (Spec B) des fonctions rationnelles sur Spec B. Les morphismes structuraux $P^{\prime \prime} \rightarrow P^{\prime}$ et $P^{\prime} \rightarrow P$ sont finis, leur composé $\theta: P^{\prime \prime} \rightarrow P$ aussi, et on a des carrés cartésiens
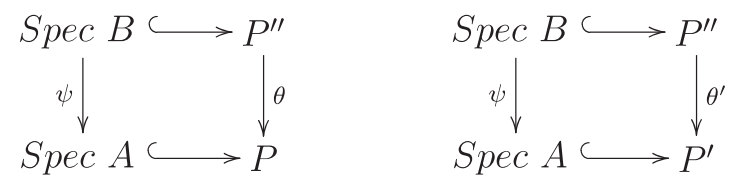

où les flèches horizontales sont des immersions ouvertes fournissant par passage aux séparés complétés des carrés cartésiens

$$
\begin{aligned}
& \begin{array}{cc}
\mathcal{S}^{\prime}:=\operatorname{Spf}_{\hat{B}} \stackrel{j^{\prime}}{\longleftrightarrow} & \hat{P}^{\prime \prime}=: \overline{\mathcal{S}^{\prime}} \\
& \downarrow \hat{\theta}
\end{array} \\
& \mathcal{S}:=\operatorname{Spf} \hat{A} \underset{\tilde{j}}{\longrightarrow} \hat{P}=: \hat{\mathcal{S}}
\end{aligned}
$$

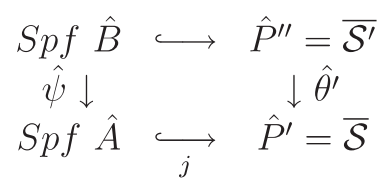

où $\hat{\psi}$ est un relèvement fini étale de $\varphi, \hat{\theta}$ et $\widehat{\theta}^{\prime}$ sont finis, $\widehat{P}^{\prime}$ est normal et les flèches horizontales sont des immersions ouvertes.

De plus $\mathcal{O}_{\widehat{P^{\prime \prime}}}$ est la fermeture intégrale de $\mathcal{O}_{\widehat{P}}\left(\right.$ resp. de $\left.\mathcal{O}_{\widehat{P^{\prime}}}\right)$ dans $\mathcal{O}_{\mathcal{S}^{\prime}}$ [EGA II, (6.3.2)]. 
Enfin, $\hat{\theta}$ (resp. $\left.\widehat{\theta}^{\prime}\right)$ est plat si et seulement si sa réduction modulo I est plate.

Donnons tout de suite un corollaire et sa démonstration avant de prouver le théorème.

Corollaire (3.1.2). Soit $\mathcal{V}$ un anneau de valuation discrète complet de caractéristique zéro, d'idéal maximal I et de corps résiduel k. Si $A_{0}$ est une $k$-algèbre lisse et $\varphi:$ Spec $B_{0} \rightarrow$ Spec $A_{0}$ est un morphisme fini étale, alors $i l$ existe une $\mathcal{V}$-algèbre lisse $A$ et un morphisme fini $\psi:$ Spec $B \rightarrow$ Spec $A$ relevant $\varphi$ et satisfaisant aux propriétés du théorème (3.1.1).

DÉmonstration du COROLlaire (3.1.2). L'existence d'une $\mathcal{V}$-algèbre lisse $A$ relevant $A_{0}$ résulte du théorème 6 de Elkik [E $\ell$ ]. Le $\mathcal{V}$ du corollaire est régulier [EGA $O_{I V}$, (17.1.4) (ii)] et excellent [EGA IV, (7.8.3)] : comme $A$ est lisse sur $\mathcal{V}, A$ est régulier [EGA IV, (17.5.8)] et excellent [EGA IV, (7.8.3)]. Il suffit alors d'appliquer le théorème (3.1.1).

\section{Démonstration du théorème (3.1.1).}

(1) et (2). D'après [EGA IV, (18.3.2)] il existe une $\hat{A}$-algèbre finie étale $C$ telle que $\operatorname{Spec} C \rightarrow \operatorname{Spec} \hat{A}$ relève $\varphi$. Puisque $A$ est noethérien normal on peut décomposer Spec $A$ en somme de ses composantes connexes $\coprod_{i}$ Spec $A_{i}$, avec $A_{i}$ intégralement clos, et $\coprod_{i}$ Spec $\hat{A}_{i}$ est une décomposition

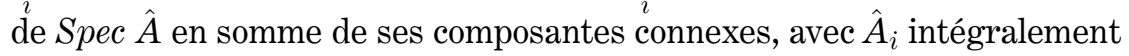
clos [prop (1.7) (4) (iv)]: $C$ est aussi normal noethérien et on le décompose de même. Comme $S p e c C \rightarrow \operatorname{Spec} \hat{A}$ est fini et plat, on est ramené au cas où ce morphisme est surjectif avec $C$ et $\hat{A}$ intégralement clos.

Soient $L$ le corps des fractions de $\hat{A}$ et $L_{1}$ celui de $C$ : d'après [EGA II, (6.1.8)] $L \hookrightarrow L_{1}$ est une extension finie de corps de caractéristique nulle (donc l'extension est séparable) et $C$ est la fermeture intégrale de $\hat{A}$ dans $L_{1}$. Par le théorème de l'élément primitif il existe $x \in L_{1}$ tel que $L_{1}=L[x]$ : $x$ est séparable sur $L$ [Bour, A V, prop 6 p. 38], son polynôme minimal $f(X) \in L[X]$ est séparable [Bour, A V, prop 5 p. 38], donc $f \wedge f^{\prime}=1$ dans $L[X]$ [Bour, A V, prop 3 p. 36]; appliquant Bézout dans $L[X]$, il existe $g_{1} \in \hat{A}$ tel que $f, f^{\prime} \in(\hat{A})_{g_{1}}[X]$ et tel qu'il existe $u, v \in(\hat{A})_{g_{1}}[X]$ vérifiant l'identité $u f+v f^{\prime}=1$ dans $(\hat{A})_{g_{1}}[X]$. Ainsi le morphisme canonique

$$
\mu:(\hat{A})_{g_{1}} \longrightarrow(\hat{A})_{g_{1}}[X] /(f)
$$


est fini étale [Mi, I, 3.4] et s'insère dans le carré cocartésien

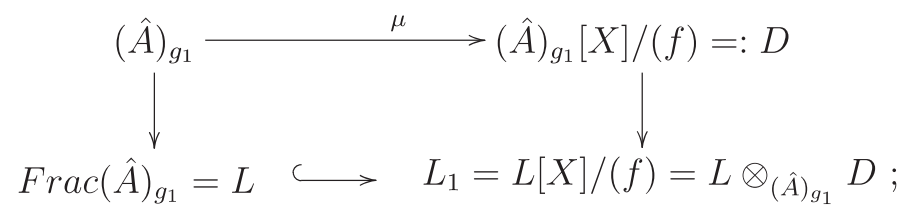

par suite $\mu$ est fidèlement plat puisqu'il est injectif. La platitude de $\mu$ fournit l'injectivité de $D \hookrightarrow L_{1}$, donc $D$ est intègre (de corps des fractions $L_{1}$ ) et normal [EGA IV, (17.5.7)], done intégralement clos; done $D$ est la fermeture intégrale de $(\hat{A})_{g_{1}}$ dans $L_{1}$. Par changement de base, $\mu$ fournit le morphisme fini étale fidèlement plat

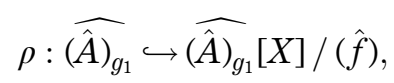

où $\hat{f}$ est l'image de $f$ dans $\widehat{(\hat{A})_{g_{1}}}[X]$.

Soit $g_{2} \in A$ un relèvement de $\left(g_{1} \bmod I\right) \in A_{0}$ : comme $\left(\widehat{\hat{A})_{g_{1}}}\right.$ est une $A$ algèbre formellement étale par les topologies $I$-adiques il existe un unique $A$ - morphisme (en fait un $\hat{A}$-morphisme)

$$
v: \widehat{(\hat{A})_{g_{1}}} \longrightarrow\left(\widehat{A_{g_{2}}}\right)
$$

relevant l'identité de $A_{g_{2}} / I A_{g_{2}}$ et $v$ est un isomorphisme [EGA $O_{I}$, (6.6.21)]. Notons $f_{1}(X) \in A_{g_{2}}[X]$ un polynôme unitaire relevant

$$
f(X) \bmod I \in(\hat{A})_{g_{1}}[X] / I(\hat{A})_{g_{1}}[X] \simeq A_{g_{2}}[X] / I A_{g_{2}}[X] ;
$$

alors, si $\hat{f}_{1}$ désigne l'image de $f_{1}$ dans $\left(\widehat{A_{g_{2}}}\right)[X],\left(\widehat{A_{g_{2}}}\right)[X] /\left(\hat{f}_{1}\right)$ est fini et plat $\operatorname{sur}\left(\widehat{A_{g_{2}}}\right)$ car $\hat{f}_{1}$ est unitaire [Mi, I, 2.6 (a)]. Comme $\rho$ est fini étale, que $D$ et $\widehat{A_{g_{2}}}[X] /\left(\hat{f}_{1}\right)$ ont même réduction $\bmod I$ et que $\widehat{(\widehat{A})_{g_{1}}}$ s'identifie à $\widehat{A_{g_{2}}}$ via $v$, il existe un unique $\widehat{A_{g_{2}}}$-morphisme

$$
\widehat{A_{g_{2}}}[X] /(v(\hat{f})) \rightarrow \widehat{A_{g_{2}}}[X] /\left(\hat{f}_{1}\right)
$$

qui est un isomorphisme [EGA $O_{I},(6.6 .21)$ ] relevant l'identité de $D / I D$. Puisqu'on a des injections

$$
\begin{gathered}
(\hat{A})_{g_{1}} \hookrightarrow(\hat{A})_{g_{1}, T} \hookrightarrow\left(\widehat{\hat{A})_{g_{1}, T}}\right)=\left(\widehat{\hat{A})_{g_{1}}}\right), \\
A_{g_{2}} \hookrightarrow A_{g_{2} T} \hookrightarrow\left(\widehat{A_{g_{2}}, T}\right)=\widehat{A_{g_{2}}}
\end{gathered}
$$

et que $f$ et $f_{1}$ sont unitaires on en déduit que

$$
v(\hat{f})=\widehat{f_{1}},
$$


i.e. que dans l'écriture $L_{1}=L[X] /(f)$ on peut supposer $f=f_{1} \in A_{g_{2}}[X]$ : ainsi il existe $g_{3} \in A$ tel que $f, f^{\prime} \in A_{g_{3}}[X]$ et tel qu'il existe $u, v \in A_{g_{3}}[X]$ vérifiant l'identité $u f+v f^{\prime}=1$ dans $A_{g_{3}}[X]$. En particulier le morphisme canonique

$$
\eta: A_{g_{3}} \rightarrow A_{g_{3}}[X] /(f)
$$

est fini étale et s’insère dans le diagramme commutatif

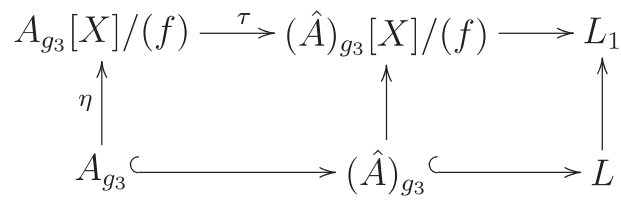

par suite $\eta$ est injectif, donc fidèlement plat. Par le même raisonnement que ci-dessus pour $D=(\hat{A})_{g_{1}}[X] /(f)$ on montre que $(\hat{A})_{g_{3}}[X] /(f)$ est intègre et intégralement clos de corps des fractions $L_{1}$. La platitude de $\eta$ fournit l'injectivité de $\tau$ : donc $A_{g_{3}}[X] /(f)$ est intègre de corps des fractions noté $K_{1}$; on notera $K$ le corps des fractions de $A$. On sait par [EGA IV, (18.10.12)] que $K_{1}$ est une extension finie étale de $K$ et que $A_{g_{3}}[X] /(f)$ est la fermeture intégrale de $A_{g_{3}}$ dans $K_{1}$ : comme $f$ est irréductible dans $L[X]$, il l'est aussi dans $K[X] \subset L[X]$, d'où $K_{1}=K[X] /(f)$.

Comme $A$ est excellent, il est universellement japonais [EGA IV, (7.8.3) (vi)] : la fermeture intégrale $B$ de $A$ dans $K_{1}=K[X] /(f)$ est une $A$-algèbre finie, Spec $B \rightarrow$ Spec $A$ est surjectif et $B_{g_{3}}=A_{g_{3}}[X] /(f) ; B$ est aussi la fermeture intégrale de $A$ dans $A_{g_{3}}[X] /(f), B_{T}$ est la fermeture intégrale de $A_{T}$ dans $A_{g_{3, T}}[X] /(f)$, et le corps des fractions de $B$ est $K_{1}$ (donc $B$ est intégralement clos). Comme Spec $\hat{A} \rightarrow$ Spec $A$ est un morphisme régulier [EGA IV, (7.8.3) (v)], done normal, la fermeture intégrale de $\hat{A}$ dans $(\hat{A})_{g_{3}}[X] /(f)$ est égale à $\hat{B}=B \otimes_{A} \hat{A}\left[\operatorname{EGA~IV,~(6.14.4)]:~or~}(\hat{A})_{g_{3}}[X] /(f)\right.$ est intégralement clos; donc $\hat{B}$ est la fermeture intégrale de $\hat{A}$ dans $L_{1}$, d'où $\hat{B}=C$. On en déduit que $B$ et $C$ ont même réduction mod $I$, d'où l'existence du morphisme fini

$$
\psi: \operatorname{Spec} B \rightarrow \operatorname{Spec} A
$$

relevant $\varphi$ et il suffit de prendre $g=g_{3}, g_{0}=g \bmod I$.

De plus Spec $\hat{A}=\operatorname{Spec} \widehat{A_{T}} \rightarrow \operatorname{Spec} A_{T}$ est un morphisme normal puisque $A_{T}$ est excellent: par suite la fermeture intégrale de $\hat{A}=\widehat{A_{T}}$ dans $(\hat{A})_{g_{3}}[X] /(f)$, qu'on sait être égale à $C=\hat{B}$, est aussi égale à $B_{T} \otimes_{A_{T}} \hat{A}=\widehat{B_{T}}=\hat{B}$ [EGA IV, (6.14.4)]. Par passage aux séparés complétés $\psi$ induit

$$
\hat{\psi}=\operatorname{Spec} \hat{B} \rightarrow \operatorname{Spec} \hat{A}
$$


qui s’identifie à notre morphisme fini étale

$$
\text { Spec } C \rightarrow \operatorname{Spec} \hat{A} ;
$$

par fidèle platitude de $\hat{A}$ sur $A_{T}$ le morphisme

$$
\psi_{T}=\operatorname{Spec} B_{T} \rightarrow \operatorname{Spec} A_{T}
$$

est donc fini étale et c'est clairement un relèvement de $\varphi: S^{\prime} \rightarrow \operatorname{Spec} A_{0}$.

(3) On se ramène à $A$ et $B$ intégralement clos comme en (1) dont on reprend les notations. Le schéma $P$ est intègre, car on a supposé $A$ intègre [EGA I, (6.10.5)], d'où $\mathcal{R}(P)=\mathcal{R}(\operatorname{Spec} A)=$ Frac $A=K$ est un corps [EGA I, (8.1.5)]. Le schéma $P$ est excellent, donc pour chaque ouvert $U=\operatorname{Spec} R \subset P, R$ est japonais [EGA IV, (7.8.3)]: ainsi la fermeture intégrale $P^{\prime}$ (resp. $\left.P^{\prime \prime}\right)$ de $P$ dans $\mathcal{R}($ Spec $A)=K$ (resp. dans $\left.\mathcal{R}(\operatorname{Spec} B)=K_{1}=K[X] /(f)\right)$ est un $P$-schéma fini et on a $\mathcal{R}\left(P^{\prime}\right)=K$ (resp. $\mathcal{R}\left(P^{\prime \prime}\right)=K_{1}$ ) [EGA II, (6.3.7)]: évidemment $P^{\prime \prime}$ est aussi la fermeture intégrale de $P^{\prime}$ dans $K_{1}$ et $P^{\prime \prime} \rightarrow P^{\prime}$ est fini. De plus $P^{\prime}$ est intégralement clos car noethérien normal et intègre [EGA II, (6.3.8)]; comme $A$ est intégralement clos, $P^{\prime}$ est aussi la fermeture intégrale de $P$ dans Spec $A$ : on a donc une immersion ouverte

$$
\operatorname{Spec} A \hookrightarrow P^{\prime}
$$

[R, cor 2, p. 42]. Par [EGA II, Rq entre (6.3.4) et (6.3.5)] ou [EGA IV, (6.14.4)] les carrés
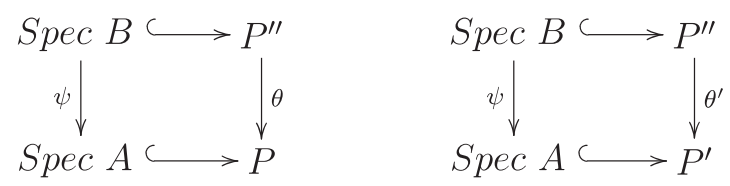

sont cartésiens.

On conclut la démonstration du théorème (3.1.1) par passage aux séparés complétés : que $\mathcal{O}_{\widehat{P^{\prime \prime}}}$ soit la fermeture intégrale de $\mathcal{O}_{\hat{P}}$ (resp. $\mathcal{O}_{\widehat{P}}$ ) dans $\mathcal{O}_{\mathcal{S}^{\prime}}$ résulte de [EGA IV, (6.14.4)] compte tenu du fait que $\hat{P} \rightarrow P$ est un morphisme normal, car $P$ est excellent. De même $\widehat{P}^{\prime} \rightarrow P^{\prime}$ est normal : ainsi $\widehat{P}^{\prime}$ est normal car $P^{\prime}$ l'est [prop (1.7)].

Il nous reste à montrer que si $\hat{\theta} \bmod I\left(\operatorname{resp} . \widehat{\theta}^{\prime} \bmod I\right)$ est plat, alors $\hat{\theta}$ (resp. $\widehat{\theta}^{\prime}$ ) est plat: faisons la démonstration pour $\hat{\theta}$. Comme ci-dessus on peut supposer $P$ intègre et se limiter à un ouvert affine $V=\operatorname{Spec} R$ de $P$ : alors l'ensemble $U$ des points de $V$ tels que la restriction de $\theta$ à $\theta^{-1}(U)$ soit plate est un ouvert non vide de $V$ [EGA IV, (11.1.1), (2.4.6), (6.9.1)] et $U$ 
contient la réduction $V_{0}$ de $V$ modulo $I$ par hypothèse. En posant: $\tilde{U}:=U \times_{V}$ Spec $\hat{R}$ et $\tilde{P}^{\prime \prime}:=\tilde{U} \times_{P} P^{\prime \prime}$, soit $\tilde{\theta}: \tilde{P}^{\prime \prime} \rightarrow \tilde{U}$ l'image inverse de $\theta$ par le changement de base $\tilde{U} \rightarrow P: \tilde{\theta}$ est plat. Or $\tilde{U}$ est un ouvert de Spec $\hat{R}$ qui contient $V_{0}$, donc $\tilde{U}=S p e c \hat{R}$ via [EGA IV, (18.5.4.3)] car (Spec $\hat{R}, V_{0}$ ) est un couple hensélien [Et 1, théo 3]. Par passage aux complétés formels, $\hat{\theta}: \widehat{P^{\prime \prime}} \rightarrow \hat{P}$ est plat.

Dans le théorème qui suit on particularise les hypothèses faites sur l'anneau $\mathcal{V}$ dans le théorème (3.1.1) ce qui permet d'étendre celui-ci du cas "fini étale" au cas "fini":

THÉORÈme (3.1.3). Soient $\mathcal{V}$ un anneau excellent normal de caractéristique zéro, $I \subset \mathcal{V}$ un idéal et $\mathcal{V}_{0}=\mathcal{V} / I$ tel que $\left(\mathcal{V}, \mathcal{V}_{0}\right)$ soit un couple hensélien au sens de [EGA IV, (18.5.5)]. Soient $A_{0}$ et $C_{0}$ deux $\mathcal{V}_{0}$-algèbres lisses et

$$
\varphi_{0}: A_{0} \rightarrow C_{0}
$$

un $\mathcal{V}_{0}$-morphisme fini (resp. fini et plat; resp. fini et fidèlement plat; resp. fini étale); fixons deux $\mathcal{V}$-algèbres lisses $A$ et $C$ relevant respectivement $A_{0}$ et $C_{0}$ et notons $\hat{A}, \hat{C}$ leurs séparés complétés I-adiques.

Alors

(1) Il existe un $\mathcal{V}$-morphisme

$$
\varphi: \hat{A} \rightarrow \hat{C}
$$

fini (resp. fini et plat; resp. fini et fidèlement plat; resp. fini étale) relevant $\varphi_{0}$.

(2) (i) Il existe une $\mathcal{V}$-algèbre de type fini $B$ normale (resp. intégralement close si A l'est) relevant $C_{0}$, un $\mathcal{V}$-morphisme fini

$$
\psi: A \rightarrow B
$$

relevant $\varphi_{0}$ et un $\mathcal{V}$-isomorphisme $\hat{C} \simeq \hat{B}$ s'insérant dans un triangle commutatif

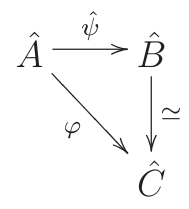

(ii) De plus les $\mathcal{V}$-morphismes

$$
\psi_{T}: A_{T} \rightarrow B_{T} \text { et } \psi^{\dagger}=\psi \otimes_{A} A^{\dagger}: A^{\dagger} \rightarrow B^{\dagger} \simeq B \otimes_{A} A^{\dagger}
$$


sont finis (resp. finis et plats; resp. finis et fidèlement plats; resp. finis étales) et relèvent $\varphi_{0}$; les morphismes $\widehat{\psi}_{T}$ et $\widehat{\psi}^{\dagger}$ s'identifient à $\hat{\psi}$.

(3) Fixons de plus une présentation de la $\mathcal{V}$-algèbre $A$

$$
A=\mathcal{V}\left[t_{1}, \ldots, t_{n}\right] /\left(f_{1}, \ldots, f_{r}\right)
$$

et reprenons les notations du (3) du théorème (3.1.1). On a les mêmes carrés cartésiens, mais où cette fois $\hat{\psi}$ est un relèvement fini (resp. fini et plat; resp. fini et fidèlement plat; resp. fini étale) de $\varphi_{0}$ et où $\theta, \theta^{\prime}, \hat{\theta}, \widehat{\theta^{\prime}}$ sont finis. De plus $\hat{\theta}$ (resp. $\widehat{\theta}^{\prime}$ ) est plat si et seulement si sa réduction modulo I est plate.

\section{DÉMONSTRATION DE 3.1.3.}

(1) L'existence de $A$ et $C$ résulte du théorème 6 de Elkik [E $\ell$ ] et celle de $\varphi$ se déduit de la lissité formelle de $\hat{A}$ sur $\mathcal{V}$ : donc $\varphi$ est un morphisme fini [Bour, AC III, § $2, \mathrm{n}^{0} 11$, prop 14].

On conclut comme dans le théorème 17 de [Et 1].

(2) et (3) Décomposant les schémas normaux Spec A, Spec C en somme de leurs composantes connexes, on peut supposer $A$ et $C$ intégralement clos: alors $\hat{A}$ et $\hat{C}$ sont aussi intégralement clos [prop (1.7) (4) (iv)], car $A$ et $C$ sont excellents. Le théorème de platitude générique [EGA IV, (6.9.1)] prouve alors la surjectivité du morphisme fini $\operatorname{Spec}(\varphi): \operatorname{Spec} \hat{C} \rightarrow \operatorname{Spec} \hat{A}$.

Soient $L$ le corps des fractions de $\hat{A}$ et $L_{1}$ celui de $\hat{C}$ : la suite de la démonstration est alors identique à celle du théorème (3.1.1).

Corollaire (3.1.4). Avec $\mathcal{V}$ comme dans le théorème (3.1.3) fixons une $\mathcal{V}$-algèbre lisse $A$. Notons $\mathcal{C}_{f}^{\dagger}$ (resp. $\mathcal{C}_{f p}^{\dagger} ;$ resp. $\mathcal{C}_{f f p}^{\dagger}$ resp. $\mathcal{C}_{\text {fet }}^{\dagger}$ ) la catégorie des $A^{\dagger}$-algèbres finies $B$ (resp. finies et plates; resp. finies et fidèlement plates; resp. finies étales) telles que B soit formellement lisse sur $\mathcal{V}$ pour la topologie I-adique: on notera $\hat{\mathcal{C}}$ (resp. $\tilde{\mathcal{C}}$ resp. $\stackrel{\mathcal{C}}{\text { ) les catégories }}$ analogues obtenues en remplaçant $A^{\dagger}$ par $\hat{A}$ (resp. par $\tilde{A}$; resp. par $A_{0}$ ); ici on a omis les indices " $f$ ", "fp" .... Alors

(1) Le foncteur

$$
\mathcal{F}: \mathcal{B} \mapsto \mathcal{B} \otimes_{A^{\dagger}} \hat{A}\left(\operatorname{resp} . \mathcal{F}: \mathcal{B} \mapsto \mathcal{B} \otimes_{\tilde{A}} A^{\dagger}\right)
$$

est une équivalence de catégories de la catégorie $\mathcal{C}_{f}^{\dagger}$ (resp. $\tilde{C}_{f}$ ) dans la catégorie $\hat{\mathcal{C}}_{f}\left(\right.$ resp. $\left.\mathcal{C}_{f}^{\dagger}\right)$ : on a les mêmes résultats avec les indices $f p, f f p$ et fét. 
(2) Un foncteur quasi-inverse de $\mathcal{F}$ est fourni par le foncteur $\mathcal{G}$ qui à une $\hat{A}$-algèbre (resp. $A^{\dagger}$-algèbre) finie $\mathcal{D}$ associe la fermeture intégrale de $A^{\dagger}$ (resp. $\left.\tilde{A}\right)$ dans $\mathcal{D}$.

(3) (i) Le foncteur

$$
\tilde{\mathcal{H}}\left(\text { resp. } \mathcal{H}^{\dagger} ; \operatorname{resp} . \mathcal{H}^{\wedge}\right): \mathcal{B} \mapsto \mathcal{B} / I \mathcal{B}
$$

est un foncteur plein et essentiellement surjectif de la catégorie $\tilde{\mathcal{C}_{f}}$ (resp. $\mathcal{C}_{f}^{\dagger}$ resp. $\hat{\mathcal{C}}_{f}$ ) dans la catégorie $\stackrel{\circ}{f}_{f}$ : on a les mêmes résultats avec les indices fp et ffp.

(ii) Le foncteur

$$
\mathcal{H}_{e t}: \mathcal{B} \mapsto \mathcal{B} / I \mathcal{B}
$$

est une équivalence de catégories de la catégorie $\tilde{\mathcal{C}}_{\text {fét }}$ (resp. $\mathcal{C}_{\text {fét }}^{\dagger}$; resp. $\hat{\mathcal{C}}_{\text {fét }}$ ) dans la catégorie $\stackrel{\circ}{\text { fét }}_{\text {. }}$

DÉmonstration. Le (3) (ii) est ici pour mémoire car montré dans [théo 2.4].

(1) et (2). On fait la démonstration pour l'indice " $f$ ", et $\mathcal{F}: \mathcal{C}_{f}^{\dagger} \rightarrow \hat{\mathcal{C}}_{f}$, les autres étant analogues.

Le foncteur $\mathcal{F}$ est fidèle d'après [EGA IV (2.2.16)].

Prouvons que $\mathcal{F}$ est essentiellement surjectif. Soit: $\varphi: \hat{A} \rightarrow C$ un objet de $\hat{\mathcal{C}_{f}}$; la réduction $\bmod I, C_{0}$, de $C$ est une $\mathcal{V}_{0}$-algèbre lisse que l'on relève par le théorème de Elkik en une $\mathcal{V}$-algèbre lisse $D$ : par lissité formelle de $C$ sur $\mathcal{V}$ il existe un $\mathcal{V}$-morphisme $\hat{\theta}_{1}: C \rightarrow \hat{D}$ et c'est un isomorphisme; comme le diagramme suivant commute:

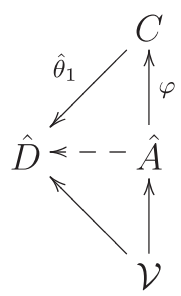

on peut voir $\hat{\theta}_{1}$ comme un $\hat{A}$-isomorphisme au moyen de la flèche en pointillé $\hat{\theta}_{1} \circ \varphi$. Le théorème (3.1.3) fournit alors une $A$-algèbre finie $B, \psi: A \rightarrow B$, et un $\hat{A}$-isomorphisme

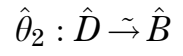

s’insérant dans le diagramme commutatif 


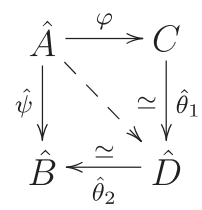

donc $\varphi \stackrel{\sim}{\rightarrow} \mathcal{F}\left(\psi^{\dagger}: A^{\dagger} \rightarrow B^{\dagger}\right)$ et $\mathcal{F}$ est essentiellement surjectif.

Prouvons que $\mathcal{F}$ est plein. Soient $\varphi: \hat{A} \rightarrow C$ et $\varphi^{\prime}: \hat{A} \rightarrow C^{\prime}$ deux objets de $\hat{\mathcal{C}}_{f}$ et $\lambda: C \rightarrow C^{\prime}$ un $\hat{A}$-morphisme. On vient de montrer qu'il existe des morphismes finis $\psi: A \rightarrow B, \psi^{\prime}: A \rightarrow B^{\prime}$ s'insérant dans le diagramme commutatif

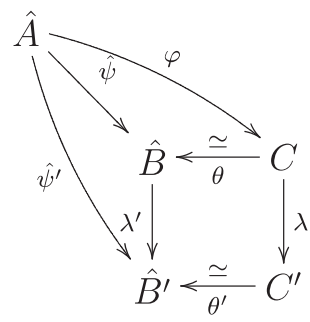

où l'on a posé $\lambda^{\prime}=\theta^{\prime} \circ \lambda \circ \theta^{-1}$. Il s'agit de trouver un $A^{\dagger}$-morphisme $\lambda^{\dagger}: B^{\dagger} \rightarrow B^{\prime \dagger}$ induisant $\lambda^{\prime}$ par passage aux complétés.

Comme dans le théorème (3.1.3) on se ramène au cas où $A, \hat{A}, C$ et $C^{\prime}$ sont intégralement clos. On a vu dans la démonstration du théorème (3.1.3) (semblable à celle de (3.1.1)) qu'il existe $a \in A$ et $f, g \in A_{a}[X]$ tels que $\psi_{a}$ et $\psi_{a}^{\prime}$ soient finis étales et s'identifient aux morphismes canoniques :

$$
\begin{gathered}
\psi_{a}: A_{a} \rightarrow B_{a}=A_{a}[X] /(f) \\
\psi_{a}^{\prime}: A_{a} \rightarrow B_{a}^{\prime}=A_{a}[X] /(g) ;
\end{gathered}
$$

$\hat{\psi}_{a}, \hat{\psi_{a}^{\prime}}$ sont finis étales et s'identifient à

$$
\begin{gathered}
(\hat{\psi})_{a}:(\hat{A})_{a} \rightarrow(\hat{B})_{a}=(\hat{A})_{a}[X] /(f) \\
\left(\hat{\psi^{\prime}}\right)_{a}:(\hat{A})_{a} \rightarrow\left(\hat{B}^{\prime}\right)_{a}=(\hat{A})_{a}[X] /(g)
\end{gathered}
$$

et font commuter le triangle dont les flèches sont finies étales

$$
\begin{array}{r}
\widehat{\left(A_{a}\right)}=\widehat{\left((\hat{A})_{a}\right)} \stackrel{\widehat{\left.(\hat{\psi})_{a}\right)}}{\longrightarrow} \widehat{\left((\hat{B})_{a}\right)}=\widehat{\left(B_{a}\right)} \\
\widehat{\left.\left(\hat{(}^{\prime}\right)_{a}\right)}=\widehat{\left.\left(\hat{\psi}^{\prime}\right)_{a}\right)}=\widehat{\left(\widehat{\lambda_{a}^{\prime}}\right)}
\end{array}
$$


Par l'équivalence de (3) (ii) il existe un morphisme $\rho$ fini étale, qui relève $\left(\widehat{\lambda_{a}^{\prime}}\right)$ et fait commuter le triangle

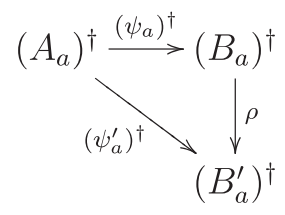

D'après la proposition 2 de [Et 2] on a les égalités

$$
A^{\dagger}=\left(A_{a}\right)^{\dagger} \cap \hat{A}, \quad B^{\dagger}=\left(B_{a}\right)^{\dagger} \cap \hat{B} \quad, \quad B^{\prime \dagger}=\left(B_{a}^{\prime}\right)^{\dagger} \cap \hat{B}^{\prime} ;
$$

comme $\rho$ et $\lambda^{\prime}$ induisent tous deux $\left(\widehat{\lambda_{a}^{\prime}}\right)$, la restriction de $\rho$ et $\lambda^{\prime}$ à $B^{\dagger}=\left(B_{a}\right)^{\dagger} \cap \hat{B}$ fournit le $A^{\dagger}$-morphisme cherché

$$
\lambda^{\dagger}: B^{\dagger} \rightarrow B^{\prime \dagger} .
$$

(2) Se démontre comme le (2) (ii) du théorème (2.4).

(3) (i) Il suffit de le prouver pour $\mathcal{H}^{\wedge}$.

On prouve que $\mathcal{H}^{\wedge}$ est essentiellement surjectif par une méthode analogue à celle utilisée pour $\mathcal{F}$ dans le (1) : étant donnée une $A_{0}$-algèbre finie $B_{0}$, on trouve une $A$-algèbre finie $B$ relevant $B_{0}$ et donc $\hat{B}$ est une $\hat{A}$-algèbre finie répondant à la question.

Pour montrer que $\mathcal{H}^{\wedge}$ est plein on part d'un $A_{0}$-morphisme $\lambda_{0}: B_{0} \rightarrow B_{0}^{\prime}$; avec $B$ et $B^{\prime}$ comme ci-dessus et lissité formelle de $\hat{B}$ sur $\mathcal{V}$ on en déduit un $\mathcal{V}$ morphisme (en fait un $\hat{A}$-morphisme) $\lambda: \hat{B} \rightarrow \hat{B}^{\prime}$ qui relève $\lambda_{0}$.

Corollaire (3.1.5). Avec les hypothèses et notations du (3) du théorème (3.1.3), supposons de plus donné un carré cartésien de $\mathcal{V}$-schémas formels

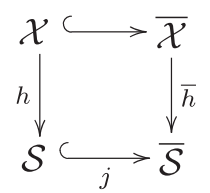

où $\bar{h}$ est propre. Alors le $\mathcal{V}$-schéma formel $\mathcal{X}^{\prime}$ défini par le produit fibré

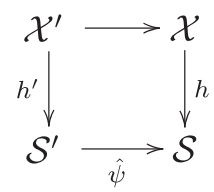


admet une compactification $\overline{\mathcal{X}^{\prime}}$ définie par le cube à faces cartésiennes

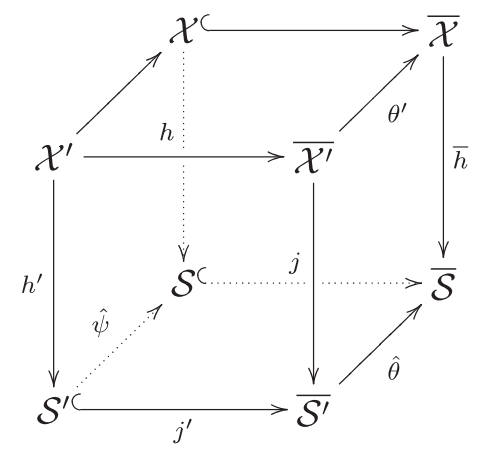

où $\hat{\theta}$ est fini.

De plus on a les mêmes résultats en remplaçant $\overline{\mathcal{S}}$ par $\tilde{\mathcal{S}}$.

DÉmonstration. Comme $j^{\prime}$ est une immersion ouverte il en est de même de $\mathcal{X}^{\prime} \rightarrow \overline{\mathcal{X}^{\prime}}$. De plus $\overline{\mathcal{X}^{\prime}} \rightarrow \overline{\mathcal{X}}$ est fini puisque $\hat{\theta}$ l'est [théo (3.1.3) (3)]; d'où le corollaire.

Corollaire (3.1.6). Avec les hypothèses et notations du théorème (3.1.3), supposons de plus $\varphi_{0}$ fini étale galoisien de groupe G. Alors

(1) $\hat{\psi}: \mathcal{S}^{\prime}=\operatorname{Spf} \hat{B} \rightarrow \mathcal{S}=\operatorname{Spf} \hat{A}$ est fini étale galoisien de groupe $G$.

(2) On a un carré cartésien

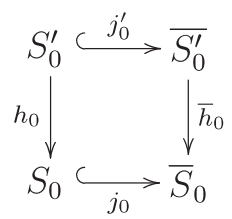

où $h_{0}=S p e c \varphi_{0}=\hat{\psi} \bmod I, \overline{S_{0}}$ et $\overline{S_{0}^{\prime}}$ sont propres sur $\mathcal{V}_{0}$ et normaux, $\overline{h_{0}}$ est fini, et $j_{0}, j_{0}^{\prime}$ sont des immersions ouvertes dominantes. De plus G agit sur $\overline{S_{0}^{\prime}}$ par $\bar{S}_{0}$-automorphismes et on a un isomorphisme

$$
\mathcal{O}_{\overline{S_{0}}} \stackrel{\sim}{\longrightarrow}\left(\bar{h}_{0 *}\left(\mathcal{O}_{\overline{S_{0}^{\prime}}}\right)\right)^{G} .
$$

Démonstration. Le (1) est classique.

Dans le (2) on prend pour $\bar{S}_{0}$ le normalisé de $\widehat{P}^{\prime} \bmod I$, et pour $\overline{S_{0}^{\prime}}$ la fermeture intégrale de $\bar{S}_{0}$ dans $S_{0}^{\prime}$; d'où le carré cartésien ci-dessus, et un carré commutatif 


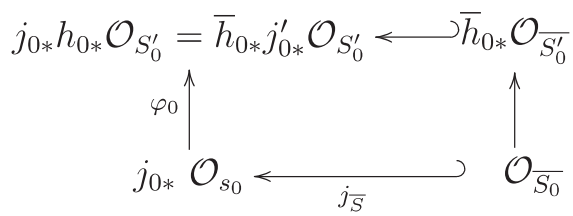

à flèches horizontales injectives.

Pour l'action de $G$ on peut supposer $S_{0}$ connexe, donc intégralement clos puisqu'il est lisse sur $\mathcal{V}_{0}$ : alors $\overline{S_{0}}$ est intègre et normal, donc $\mathcal{O}_{\bar{S}_{0}}$ est un faisceau d'anneaux intégralement clos, de corps des fractions celui de $\mathcal{O}_{S_{0}}$.

Considérons $g \in G$ et une section $x$ de $\bar{h}_{0 *} \mathcal{O}_{\overline{S_{0}^{\prime}}}$ : alors $g(x)$ est une section de $j_{0 *} h_{0 *} \mathcal{O}_{S_{0}^{\prime}}$ qui est entière sur $\mathcal{O}_{\bar{S}_{0}}$, donc $g(x)$ est en fait une section de $\bar{h}_{0 *} \mathcal{O}_{\overline{S_{0}^{\prime}}}$ car $\overline{S^{\prime}}{ }_{0}$ est la fermeture intégrale de $\bar{S}_{0}$ dans $S_{0}^{\prime}$.

Si l'on suppose de plus $x$ fixe par $G$, alors $x$ est une section de $j_{0 *} \mathcal{O}_{S_{0}}$ car $\varphi_{0}$ est galoisien de groupe $G$; or $x$ est entière sur $\mathcal{O}_{\bar{S}_{0}}$, donc $x$ est une section de $\mathcal{O}_{\bar{S}_{0}}$ puisque $\mathcal{O}_{\bar{S}_{0}}$ est intégralement clos. D'où le corollaire.

3.2 - Cas des morphismes projectifs et des intersections complètes.

THÉORÈme (3.2.1). Soient $\mathcal{V}$ un anneau excellent normal, $I \subset \mathcal{V}$ un idéal et $\mathcal{V}_{0}=\mathcal{V} /$ I tel que $\left(\mathcal{V}, \mathcal{V}_{0}\right)$ soit un couple hensélien au sens de [EGA $I V$, (18.5.5)]; on suppose $\mathcal{V}_{0}$ normal. Soient $S_{0}=S p e c A_{0}$ un $\mathcal{V}_{0}$-schéma affine et lisse, $A=\mathcal{V}\left[t_{1}, \ldots, t_{d}\right] / J$ une $\mathcal{V}$-algèbre lisse relevant $A_{0}$ et dont on a fixé une présentation et $S=$ Spec $A$ : on note $\hat{A}$ le séparé complété de $A$ pour la topologie I-adique, $A^{\dagger}$ son complété faible, $\tilde{A}$ l'hensélisé de $A$ au sens de Raynaud et $\hat{S}=$ Spec $\hat{A}, S^{\dagger}=S p e c A^{\dagger}, \tilde{S}=$ Spec $\tilde{A}$. On désigne par $\bar{S}$ l'adhérence schématique de $S$ dans $\mathbb{P}_{\mathcal{V}}^{d}$, et par $\mathcal{S}($ resp. $\overline{\mathcal{S}}$ ) le complété formel I-adique de $S$ (resp. de $\bar{S}$ ).

Soitf $: X_{0} \rightarrow S_{0}$ un $\mathcal{V}_{0}$-morphisme projectif. Alors

(1) Il existe un carré cartésien

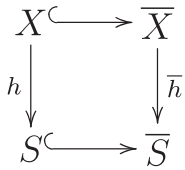

dans lequel $\bar{h}$ est projectif, $h$ est un relèvement projectif de $f$ et les flèches horizontales sont des immersions ouvertes. 
(2) Considérons le diagramme commutatif à carrés cartésiens déduit de (3.2.1.1)

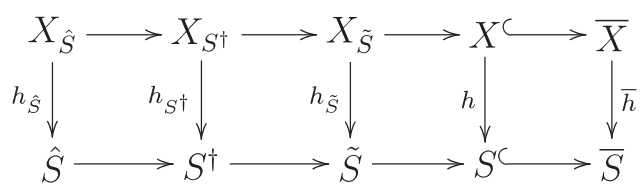

dans lequel $h, h_{\tilde{S}}, h_{S^{\dagger}}, h_{\hat{S}}$ sont des relèvements projectifs de $f$.

Alors on a équivalence entre les propriétés suivantes :

(i) $X$ est plat sur $\mathcal{V}$ et $f$ est plat.

(ii) $h_{\hat{S}}$ est plat.

(iii) $h_{S^{\dagger}}$ est plat.

(iv) $h_{\tilde{S}}$ est plat.

(3) On a équivalence entre les propriétés suivantes:

(i) $X$ est plat sur $\mathcal{V}$ et $f$ est lisse.

(ii) $h_{\hat{S}}$ est lisse.

(iii) $h_{S^{\dagger}}$ est lisse.

(iv) $h_{\tilde{S}}$ est lisse.

(4) Le carré cartésien (3.2.1.1) fournit par passage aux complétés formels un carré cartésien de $\mathcal{V}$-schémas formels

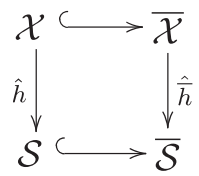

dans lequel $\hat{h}$ est un relèvement projectif de $f, \hat{\bar{h}}$ est projectif et les flèches horizontales sont des immersions ouvertes.

De plus on a équivalence entre les propriétés suivantes :

(i) $X$ est plat sur $\mathcal{V}$ et $f$ est plat (resp. $f$ est lisse).

(ii) $\hat{h}$ est plat (resp. $\hat{h}$ est lisse).

DÉmonstration. Quitte à décomposer les schémas normaux noethériens $S p e c \mathcal{V}$ et $S_{0}$ en somme de leurs composantes connexes on supposera dans toute la suite que $S p e c \mathcal{V}$ et $S_{0}$ sont connexes, donc intégralement clos.

Pourle (1). Le morphisme projectif $f$ se factorise en $f: X_{0} \stackrel{i_{0}}{\rightarrow} \mathbb{P}_{A_{0}}^{n} \stackrel{s_{0}}{\rightarrow} S_{0}=$ $=$ Spec $A_{0}$ où $i_{0}$ est une immersion fermée et $s_{0}$ est le morphisme canonique. Soient $\left(x_{0}, x_{1}, \ldots, x_{n}\right)$ les coordonnées projectives sur $\mathbb{P}_{A_{0}}^{n}$ (resp. sur $\left.\mathbb{P}_{S}^{n}\right)$ :alors $X_{0}$ est isomorphe à $\operatorname{Proj}\left(A_{0}\left[x_{0}, x_{1}, \ldots, x_{n}\right] / \mathcal{J}^{0}\right)$ pour un certain idéal homogène $\mathcal{J}^{0}$ de l'anneau noethérien $A_{0}\left[x_{0}, x_{1}, \ldots, x_{n}\right]: \mathcal{J}^{0}$ est engendré par un 
nombre fini de polynômes homogènes $f_{0}^{1}, \ldots, f_{0}^{r}$. Pour $\alpha \in\{1, \ldots, r\}$ relevons $f_{0}^{\alpha}$ en un polynôme homogène $f^{\alpha} \in A\left[x_{0}, x_{1}, \ldots, x_{n}\right]$ de même degré en relevant coefficient par coefficient de $A_{0}$ à $A$, et soit $\mathcal{J}$ l'idéal (homogène) engendré $\operatorname{par} f^{1}, \ldots, f^{r}$

$$
\mathcal{J}=\left(f^{1}, \ldots, f^{r}\right) \subset A\left[x_{0}, x_{1}, \ldots, x_{n}\right] .
$$

Désignons par

$$
i: X=\operatorname{Proj}\left(A\left[x_{0}, x_{1}, \ldots, x_{n}\right] / \mathcal{J}\right) \hookrightarrow \mathbb{P}_{S}^{n}
$$

l'immersion fermée et par $p_{S}: \mathbb{P}_{S}^{n} \rightarrow S=$ Spec $A$ la projection canonique. Alors le morphisme composé

$$
h=p_{S} \circ i: X \rightarrow S
$$

est un relèvement projectif de $f$. Notons $p_{\bar{S}}: \mathbb{P}_{\bar{S}}^{n} \rightarrow \bar{S}$ la projection canonique, $\bar{X}$ la fermeture intégrale de $\mathbb{P}_{\bar{S}}^{n}$ dans $X, \bar{i}: \bar{X} \hookrightarrow \mathbb{P}_{\bar{S}}^{n}$ l'immersion fermée et $\bar{h}=p_{\bar{S}} \circ \bar{i}: \bar{h}$ est projectif. On dispose ainsi d'un carré cartésien

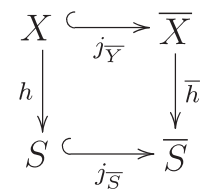

où les flèches horizontales sont des immersions ouvertes.

Pour le (2). On a le lemme suivant:

Lemme (3.2.2). On a l'équivalence:

$X$ est plat sur $\mathcal{V} \Longleftrightarrow X_{\hat{S}}$ est plat sur $\mathcal{V}$.

DÉmonstration DU LEMme. Notons $v$ le morphisme composé $X \stackrel{h}{\rightarrow} S \rightarrow$ $\rightarrow$ Spec $\mathcal{V}$ et $w$ le composé de $v$ avec le morphisme plat $X_{\hat{S}} \rightarrow X$. Si $v$ est plat, $w$ l'est. Supposons que $w$ soit plat : d'après le critère de platitude par fibres [EGA IV,(11.3.10)] $v$ est plat sur sa fibre spéciale $X_{0}$, donc audessus de $\mathcal{V}_{0}$. Or par le théorème de platitude générique [EGA IV, (6.9.1)] il existe un ouvert non vide $V$ de $S p e c \mathcal{V}$ au-dessus duquel $v$ est lisse; comme cet ouvert $V$ contient $S p e c \mathcal{V}_{0}$ d'après le raisonnement fait cidessus, il résulte de [EGA IV, (18.5.4.3)] que $V=\operatorname{Spec} \mathcal{V}$ puisque $\left(\mathcal{V}, \mathcal{V}_{0}\right)$ est un couple hensélien. D'où le lemme. 
On montre de la même façon le lemme suivant:

Lemme (3.2.3). Avec $\mathcal{V}$ comme dans le théorème (3.2.1), soit $B$ une $\mathcal{V}$-algèbre de type fini, de séparé complété I-adique noté $\hat{B}$. Alors on a l'équivalence :

$$
B \text { est plat sur } \mathcal{V} \Longleftrightarrow \hat{B} \text { est plat sur } \mathcal{V} .
$$

Par fidèle platitude des morphismes $\hat{S} \rightarrow S^{\dagger}$ et $S^{\dagger} \rightarrow \tilde{S}$ les propriétés (ii), (iii), (iv) sont équivalentes.

Supposons (ii) vérifié : alors $X_{\hat{S}}$ est plat sur $\mathcal{V}$, donc par le lemme (3.2.2) $X$ est plat sur $\mathcal{V}$ et $(i)$ est clair. Il nous reste à prouver que $(i) \Rightarrow(i i)$.

Supposons la propriété $(i)$ vérifiée. Puisque $X_{\hat{S}}$ est plat sur $\mathcal{V}$ (car $X$ est plat sur $\mathcal{V}$ ) et $f$ est plat, le critère de platitude par fibres [EGA IV,(11.3.10)] prouve que $h_{\hat{S}}$ est plat en tous les points au-dessus de $S_{0}$. Or Spec $\hat{A}$ est connexe car $S_{0}=$ Spec $A_{0}$ l'est [Et 1, cor 2 du théo 3]; comme $A$ est normal et que le morphisme $A \rightarrow \hat{A}$ est normal, car régulier [EGA IV, (7.8.3) (v)], il résulte de la [prop (1.7) 4 (ii)] que $\hat{A}$ est normal, donc que $\hat{A}$ est intégralement clos. Par le théorème de platitude générique [EGA IV, (6.9.1)] il existe un ouvert non vide $V$ de Spec $\hat{A}$ tel que la restriction $h_{V}: h_{\hat{S}}^{-1}(V) \rightarrow V$ de $h_{\hat{S}}$ soit plate. Or $V$ contient Spec $A_{0}$ d'après le raisonnement fait ci-dessus, done $V=\operatorname{Spec} \hat{A}=: \hat{S}$ puisque $\left(\hat{S}, S_{0}\right)$ est un couple hensélien [EGA IV, (18.5.4.3)].

Pour le (3). Par fidèle platitude des morphismes $\hat{S} \rightarrow S^{\dagger}$ et $S^{\dagger} \rightarrow \tilde{S}$ les propriétés $(i i),(i i i),(i v)$ sont équivalentes. Comme $(i i) \Rightarrow(i)$ est clair, il nous reste à prouver que $(i) \Rightarrow(i i)$.

Supposons la propriété $(i)$ vérifiée. D’après le $(2) h_{\hat{S}}$ est plat. Appliquons [EGA IV, (12.2.4)] au morphisme projectif et plat $h_{\hat{S}}:$ l'ouvert $W \operatorname{des} s \in \hat{S}$ où $h_{s}:\left(X_{\hat{S}}\right)_{s} \rightarrow s$ est lisse contient $S p e c A_{0}$ puisque $f$ est lisse, donc $W=\hat{S}$ là encore en utilisant le caractère hensélien du couple $\left(\hat{S}, S_{0}\right)$. Ainsi on a obtenu un relèvement projectif et lisse $h_{\hat{S}}: X_{\hat{S}} \rightarrow \hat{S}$ de $f$.

Pour le (4). Il suffit de prendre le complété formel du carré (3.2.1.1): on obtient un carré cartésien de $\mathcal{V}$-schémas formels

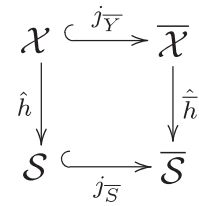


dans lequel $\hat{h}$ et $\hat{\bar{h}}$ sont projectifs. Puisque $\hat{A}$ est un anneau de Zariski et que $\hat{h}$ est le complété de $h, h_{\hat{S}}$, il résulte de [Bour, AC III, $\S 5 ; \mathrm{n}^{\circ} 4$, prop 2 , et $\mathrm{n}^{0} 2$, théo 1] que la platitude (resp. la lissité) de $\hat{h}$ équivaut à celle de $h_{\hat{S}}$; d'où l'équivalence des propriétés (i) et (ii) .

Remarques (3.2.4). Supposons que $\mathcal{V}$ est un anneau de valuation discrète complet, $I$ son idéal maximal, $k$ son corps résiduel et $\pi$ une uniformisante.

(i) L'hypothèse de platitude de $X$ sur $\mathcal{V}$ dans [(3.2.1) (2), (3) et (4)] équivaut à $\mathcal{O}_{X}$ sans $\pi$-torsion.

(ii) Lorsque $S=S p e c \mathcal{V}$, un exemple de Serre [S 1] prouve qu'il existe des cas où $X$ n'est pas plat sur $\mathcal{V}:$ dans ce cas $h$ n'est pas plat. Nous allons voir ci-dessous en (3.2.6) une condition suffisante de platitude de $h$, celle où le morphisme projectif $f$ identifie $X$ à une intersection complète dans un espace projectif (cf. définition (3.2.5)).

En nous inspirant de la définition donnée par Deligne des intersections complètes dans un fibré projectif [SGA 7, II, exp.XI, 1.4] on adoptera la définition suivante:

DÉfinition (3.2.5). Soit $f: X \rightarrow S$ un morphisme de schémas. On dira que $X$ est une intersection complète relativement à $S$ dans des espaces projectifs sur $S$ si il existe un recouvrement de $S$ par des ouverts de Zariski $S_{\alpha}, S=\bigcup_{\alpha} S_{\alpha}$ et, en désignant par $f_{\alpha}: X_{\alpha} \rightarrow S_{\alpha}$ le morphisme déduit de $f$ par le changement de base $S_{\alpha} \rightarrow S$, il existe, pour chaque $\alpha$, un couple $\left(n_{\alpha}, r_{\alpha}\right) \in \mathbb{N}^{*} \times \mathbb{N}$ et une $S_{\alpha}$-immersion fermée $i_{\alpha}$ qui factorise $f_{\alpha}$

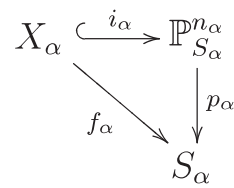

où $p_{\alpha}$ désigne la projection canonique, telle que:

(i) fibre $\grave{a}$ fibre, $i_{\alpha}$ est de codimension $r_{\alpha}$, i.e. pour tout $s=\operatorname{Spec} k(s) \in S_{\alpha}$, si $i_{\alpha, s}: X_{\alpha, s} \hookrightarrow \mathbb{P}_{s}^{n_{\alpha}}$ désigne la fibre de $i_{\alpha}$ audessus de $s$, on a $r_{\alpha}=\operatorname{codim}\left(X_{\alpha, s}, \mathbb{P}_{s}^{n_{\alpha}}\right)$

(ii) il existe un recouvrement de $S_{\alpha}$ par des ouverts de Zariski $S_{\alpha, \beta}$, $S_{\alpha}=\bigcup_{\beta} S_{\alpha, \beta}$ tels que, en désignant par 


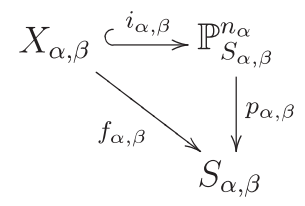

le diagramme déduit de (3.2.5.1) par le changement de base $S_{\alpha, \beta} \rightarrow S_{\alpha}$, chaque $X_{\alpha, \beta}=\operatorname{Proj}\left(\mathcal{O}_{S_{\alpha, \beta}}\left[x_{0}, x_{1}, \ldots, x_{n_{\alpha}}\right] / \mathcal{J}_{\alpha, \beta}\right)$ est l'intersection de $r_{\alpha}$ hypersurfaces (de certains degrés) au sens schématique, i.e. l'idéal $\mathcal{J}_{\alpha, \beta}$ est engendré par $r_{\alpha}$ éléments homogènes.

On dit alors que $X_{\alpha}$ est une intersection complète relativement à $S_{\alpha}$ dans $\mathbb{P}_{S_{\alpha}}^{n_{\alpha}}$ de codimension $r_{\alpha}$.

Nous allons établir le corollaire suivant du théorème (3.2.1):

Corollaire (3.2.6). Soient $\mathcal{V}$ un anneau local normal d'idéal maximal $I \subset \mathcal{V}$, complet pour la topologie I-adique, de corps résiduel $k=\mathcal{V} / I$. Soient $S_{0}$ un k-schéma lisse et séparé, $f: X_{0} \rightarrow S_{0}$ un $k$-morphisme projectif et lisse tel que $X_{0}$ est une intersection complète, relativement à $S_{0}$, dans des espaces projectifs sur $S_{0}, S_{\alpha, \beta}=S p e c A_{0}$ un $k$ schéma affine et lisse tel qu'en (3.2.5.2), $A=\mathcal{V}\left[t_{1}, \ldots, t_{d}\right] / J$ une $\mathcal{V}$-algèbre lisse relevant $A_{0}$, dont on a fixé une présentation, et $S=S p e c A$ : on note $\hat{A}$ le séparé complété de A pour la topologie I-adique, $A^{\dagger}$ son complété faible, $\tilde{A}$ l'hensélisé de $A$ au sens de Raynaud et $\hat{S}=$ Spec $\hat{A}$, $S^{\dagger}=$ Spec $A^{\dagger}, \tilde{S}=$ Spec $\tilde{A}$. On désigne par $\bar{S}$ l'adhérence schématique de $S$ dans $\mathbb{P}_{\mathcal{V}}^{d}$, et par $\mathcal{S}$ (resp. $\overline{\mathcal{S}}$ ) le complété formel I-adique de $S$ (resp. de $\bar{S}$ ).

Alors le $X$ construit en [(3.2.1)(1)] est plat sur $\mathcal{V}$ et les morphismes $h_{\tilde{S}}, h_{S^{\dagger}}, h_{\hat{S}}$ de (3.2.1.2) sont des relèvements projectifs et lisses du morphisme $f_{\alpha, \beta}: X_{\alpha, \beta} \rightarrow S_{\alpha, \beta}$ déduit de f par le changement de base $S_{\alpha, \beta} \rightarrow S_{0}$. De plus on dispose d'un diagramme tel que (3.2.1.4) dans lequel $\hat{h}$ est un relèvement projectif et lisse def.

DÉmonstration. L'anneau $\mathcal{V}$ est excellent [EGA IV, (7.8.3)(iii)]. Quitte à faire le changement de base $S_{\alpha, \beta} \rightarrow S_{0}$ on supposera dans la suite de la démonstration que $f_{\alpha, \beta}=f$. Quitte à décomposer les schémas normaux noethériens $S p e c \mathcal{V}, S_{0}$ et $X_{0}$ en somme de leurs composantes connexes on supposera dans toute la suite que $S p e c \mathcal{V}, S_{0}$ et $X_{0}$ sont connexes, donc intégralement clos. En utilisant alors les notations de la preuve de (3.2.1), avec $X_{0}=\operatorname{Proj}\left(A_{0}\left[x_{0}, x_{1}, \ldots, x_{n}\right] / \mathcal{J}^{0}\right)$, on dispose du diagramme commutatif 


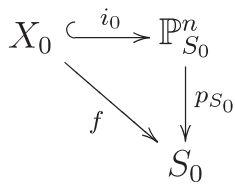

Comme $f$ est plat et $S_{0}$ connexe, pour tout $s \in S_{0}$, la dimension de $X_{0, s}=f^{-1}(s)$ est constante [H, III, cor 9.10], donc la codimension Codim $\left(X_{0, s}, \mathbb{P}_{k(s)}^{n}\right)$ aussi : notons $r$ cette dernière. Puisque $X_{0}$ est une intersection complète, relativement à $S_{0}$, dans $\mathbb{P}_{S_{0}}^{n}$, l'idéal $\mathcal{J}^{0}$ possède une famille génératrice constituée de $r$ éléments homogènes : notons ceux-ci $f_{0}^{1}, \ldots, f_{0}^{r}$, que l'on relève comme dans la preuve de (3.2.1) en $f^{1}, \ldots, f^{r}$; d'où un diagramme tel que (3.2.1.1). Notons $\mathrm{A}_{S,(j)}^{n}$, $j \in \llbracket 0, n \rrbracket$, chacun des espaces affines qui recouvrent $\mathbb{P}_{S}^{n}$ et $X_{(j)}=$ $=\operatorname{Spec}\left(A\left[x_{0}, \ldots, x_{n}\right] /\left(x_{j}-1, f^{1}, \ldots, f^{r}\right)\right)$ le schéma induit sur $\mathbb{A}_{S,(j)}^{n}$ par $X$. Il s'agit de montrer que, pour tout $j, X_{(j)}$ est plat sur $S$. Pour $j \in \llbracket 0, n \rrbracket$, notons $X_{0,(j)}$ (resp $A_{S_{0},(j)}^{n}$ ) la réduction de $X_{(j)}$ $\left(\operatorname{resp} A_{S,(j)}^{n}\right) \bmod I$ et $f_{0,(j)}^{\ell}\left(x_{0}, \ldots, x_{j-1}, x_{j+1}, \ldots, x_{n}\right)=f_{0}^{\ell}\left(x_{0}, \ldots, x_{j-1}, x_{j}=\right.$ $\left.=1, x_{j+1}, \ldots, x_{n}\right), \ell \in \llbracket 1, r \rrbracket$. Pour prouver la platitude de $X_{(j)}$ sur $S$, il suffit d'après [Mi, I, Rk $2.6(\mathrm{~d})]$ de prouver que $\left(f_{0,(j)}^{1}, \ldots, f_{0,(j)}^{r}\right)$ est une suite régulière, ce qui équivaut ici $\left[E G A 0_{I V}\right.$, (15.2.2) et (15.2.3)] à prouver qu'elle est quasi-régulière. Or $i_{0}$ est une immersion fermée régulière d'après [EGA IV, (17.12.1)], i.e., pour tout $j \in \llbracket 0, n \rrbracket$, l'idéal $\mathcal{J}_{(j)}^{0}:=\left(f_{0,(j)}^{1}, \ldots, f_{0,(j)}^{r}\right)$ est régulier [EGA IV, (16.9.2)] : donc, d'après [EGA $\left.0_{I V},(15.2 .2)\right]$ et [EGA IV, (16.1.2.2)], l'homomorphisme (ii) de [EGA IV, (16.9.3)] est bijectif. Comme $i_{0}$ est régulière, donc quasi-régulière, le faisceau conormal $\mathcal{N}_{X_{0} / P_{S_{0}}^{n}}$ est localement libre [EGA IV, (16.9.8)], i.e. pour tout $j \in \llbracket 0, n \rrbracket \mathcal{J}_{(j)}^{0} /\left(\mathcal{J}_{(j)}^{0}\right)^{2}$ est localement libre : or ici, fibre à fibre au-dessus de chaque point $s \in S_{0}$, il est de rang égal à rg $\left(\mathcal{J}_{(j)}^{0} /\left(\mathcal{J}_{(j)}^{0}\right)^{2}\right)=\operatorname{Codim}\left(X_{0, s}, \mathbb{P}_{k(s)}^{n}\right)=r$. Donc, pour tout $j \in \llbracket 0, n \rrbracket$, $\mathcal{J}_{(j)}^{0} /\left(\mathcal{J}_{(j)}^{0}\right)^{2}$ est localement libre de rang $r$. Or les images de $f_{0,(j)}^{1}, \ldots, f_{0,(j)}^{r}$ dans $\mathcal{J}_{(j)}^{0} /\left(\mathcal{J}_{(j)}^{0}\right)^{2}$ l'engendrent en tant que $\mathcal{O}_{X_{0,(j)}} / \mathcal{J}_{(j)}^{0}$-module: étant au nombre de $r$ c'en est une base [Bour, AC II, $\S 3$, cor 5 du théo 1]; ainsi [EGA IV, (16.9.3)] la suite $\left(f_{0,(j)}^{1}, \ldots, f_{0,(j)}^{r}\right)$ est quasi-régulière. Ceci achève la preuve du corollaire.

\section{RÉFÉRENCES}

[Bo] S. Bosch, A rigid analytic version of $M$. Artin's theorem on analytic equations, Math. Ann., 255 (1981), pp. 395-404. 
[Bo-Dw-R] S. Bosch - B. Dwork - PH. RoBba, Un théorème de prolongement pour des fonctions analytiques, Math. Ann., 252 (1980), pp. 165-173.

[Bour] N. BourBaki, Algèbre [A] chap. I à VII; Algèbre commutative [AC] chap. I à X.

[EGA] A. Grothendieck - J. Dieudonné, Eléments de Géométrie Algébrique: Chap. I, Springer Grundlehren 166; Chap. II, III, IV, Pub. Math. IHES n ${ }^{0}$ $8,11,17,20,24,28,32$.

[E $\ell$ R. ELKIK, Solutions d'équations à coefficients dans un anneau hensélien, Annales Scient. Ec. Norm. Sup., 4ème série, t. 6 (1973), pp. 553-604.

[Et 1] J.-Y. ETESSE, Relèvement de schémas et algèbres de Monsky-Washnitzer: théorèmes d'équivalence et de pleine fidélité, Rendiconti Sem. Mat. Univ. Padova, 107 (2002), pp. 111-138.

[Et 2] J.-Y. ETESSE, Descente étale des F-isocristaux surconvergents et rationalité des fonctions $L$ de schémas abéliens, Annales Scient. Ec. Norm. Sup., 4ème série, t. 35 (2002), pp. 575-603.

[Et 3] J.-Y. ETESSE, Images directes I: Espaces rigides analytiques et images directes en cohomologie rigide, arXiv:0910.4433; hal.00425909.

[Et 4] J.-Y. ETESSE, Images directes II: F-isocristaux convergents sur un schéma lisse, et images directes, arXiv:0910.4434; hal.00425919.

[Et 5] J.-Y. ETESSE, Images directes III: Images directes de F-isocristaux surconvergents, arXiv:0910.4435; hal.00425922.

[Et 6] J.-Y. ETESSE, Images directes et fonctions $L$ en cohomologie rigide, arXiv:0803.1580; hal.00262316.

[H] R. Hartshorne, Algebraic Geometry, Graduate Texts in Math., 52 (Springer, 1977).

[Mi] J.-S. Milne, Etale cohomology, Princeton University Press (1980).

[M-W] P. Monsky - G. Washnitzer, Formal Cohomolgy I, Annals of Math., 88, $\mathrm{n}^{\mathrm{o}} 2$ (1968), pp. 181-217.

[R] M. Raynaud, Anneaux locaux henséliens, Lecture Notes in Math., 169 (Springer, 1970).

[S] J.-P. SERRE, Exemples de variétés projectives en caractéristique p non relevables en caractéristique zéro, Proc. Nat. Acad. Sci. USA 47 (1961), pp. 108-109: Oeuvres complètes (Serre), 2, nº 50 (Springer, 1986-2000), p. 98.

[SGA 1] A. GROTHENDIECK, Revêtements étales et goupe fondamental, Lecture Notes in Math., 224 (Springer, 1971).

[SGA 7, II] P. Deligne - N. Katz, Groupes de Monodromie en Géométrie Algébrique, Lecture Notes in Math., 340 (Springer, 1973).

Manoscritto pervenuto in redazione il 15 gennaio 2009. 